CINTHIA PASSOS ASSUMPÇÃO PEDROSO

\title{
Aspectos clínicos e terapêuticos da candidíase sistêmica em UTI neonatal: estudo de 60 casos
}

Dissertação apresentada à Faculdade de Medicina da Universidade de São Paulo para obtenção do título de Mestre em Ciências

Área de concentração: Pediatria

Orientadora:

Prof $^{\mathrm{a}} \cdot \mathrm{Dr}^{\mathrm{a}}{ }^{\mathrm{a}}$ Vera Lúcia Jornada Krebs

SÃO PAULO

2005 


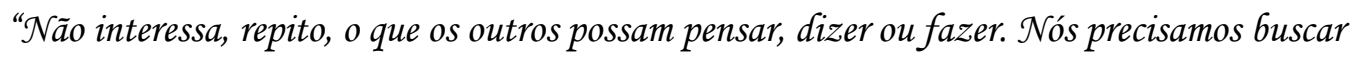
nossos limites máximos, não apenas boiar à deriva, ao sabor da correnteza, ou de má vontade apanhar uma onda e deixar-nos levar à praia. SNão. Devemos voar."

Charles R. Swindoll.

O mundo está nas mãos daqueles que têm coragem de sonhar e correr o risco de viver seus sonhos. Cada um com seu talento. Paulo Coetho 
Mestre não é quem sempre ensina, mas quem de repente aprende.

Guimarães Rosa 


\section{Dedicatória}

Aos meus pais Violeta e Jacintho, meus irmãos Claudia, Jacintho Júnior, Mário,

Márcio e Rafaela

Ao meu esposo Jocimar Carlos.

Meus filhos Lucas e Guilherme

In memorian: Meus avós Mário e Francisco

Minhas avós Íris e Raimunda

Minha avó do coração Maria Alice

A minha singela homenagem pelos ensinamentos, convívio, momentos compartilhados e a certeza que mesmo distantes estamos unidos pelas lembranças, amor e cumplicidade em tudo em nossas vidas. 
Se eu puder aliviar a aflição de uma vida,

Ou aplacar uma dor,

Ou ajudar um frágil passarinho

A retornar ao seu ninho,

$\mathcal{N} a \tilde{o}$ terei vivido em vão.

Emily Dickinson

Agradecimentos

À Deus, por sempre ter me protegido,

Aos meus pais, pelo incentivo e motivação,

À minha orientadora Profa. Dra. Vera Lúcia Jornada Krebs pela orientação, confiança,

Ao meu esposo pelo apoio e companheirismo em todos as etapas desde trabalho,

Aos meus mestres Profa . Dra Edna Maria de Albuquerque Diniz, Profa.. Maria Esther Jurferst

Ceccon e Dr. Vlisses Dória Filho, pelos ensinamentos, incentivos e sugestões;

Aos funcionários da pós graduação do Instituto da Criança HC-FMUSP: Denise, Rosimeire,

Solange, Marlene, Dóris.

Aos amigos Maria de Lourdes, Nivaldo e Milene pela colaboração, incentivo e presteza,

À Marisa Kasue Vmetsu Yoshikawa pelo seu constante auxílio, incentivo em todas as horas e colaboração com a referência bibliográfica,

Aos funcionários do Same e do arquivo pela ajuda com levantamento de prontuários,

Aos Professores de todas as disciplinas que cursei,

Aos meus amigos:Adriana, Alessandra, Ana Cristina, Ana Cristina Cruz, Ana Tereza ,Carla, Carlos, Cecília, Cristina Erico, Daniela, Elda , Eliene,Eloy, Fernanda, Francymare, Humberto, Janeide e Janete, Kátia, Marizilda, Marcos, Renata, Tarcila e a todos que compartilharam momentos quer seja de incentivo ou até mesmo na elaboração deste trabalho.

Aos meus pequenos pacientes e seus familiares.

Anseio por executar uma tarefa grande e nobre, mas é meu dever principal executar tarefas humildes como se fossem grandes e nobres. O mundo é movido não só pelos vigorosos empurrões dos seus heróis, mas também pelo conjunto de pequenos empurrões de cada trabalhador honesto. 


\title{
SUMÁRIO
}

\author{
LISTA DE ABREVIATURAS \\ LISTA DE TABELAS \\ LISTA DE GRÁFICOS \\ RESUMO \\ SUMMARY
}

INTRODUCÃOO

OBJETIVOS

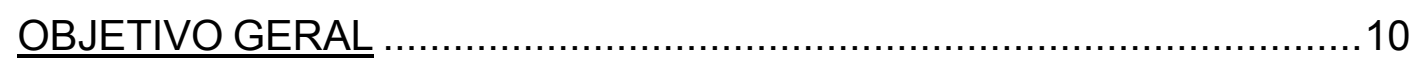



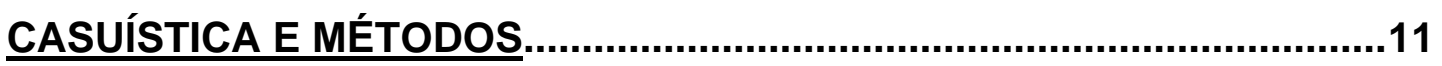



CRITÉRIOS DE INCLUSÃO E EXCLUSÃO ………….........................13

MÉTODOS

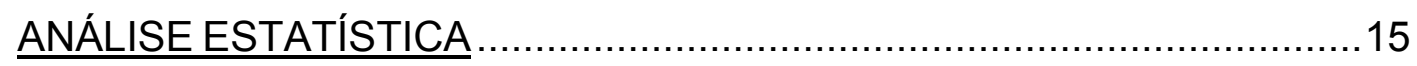

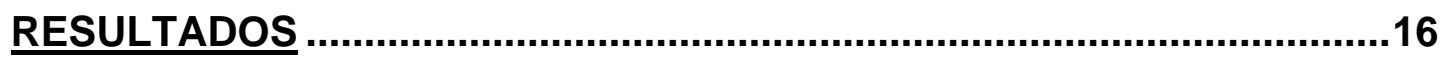

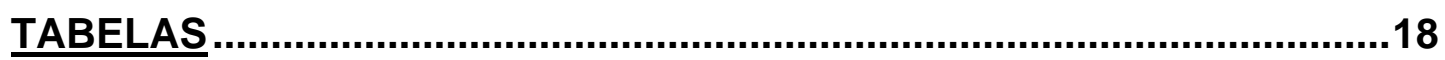

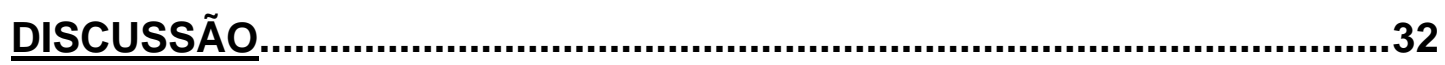

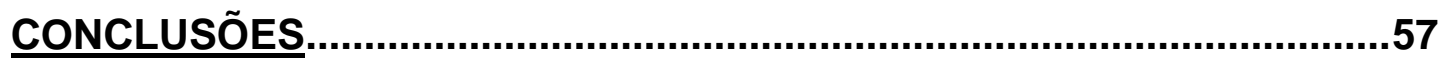

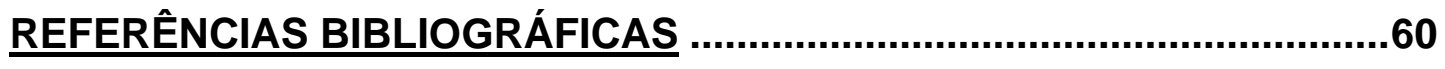




\section{Lista de Abreviaturas}

C - Candida

d-dias

F-teste exato de Fisher

RN-Recém-nascido

RNPT- Recém-nascido Pré-termo

RNT- Recém-nascido termo

Sem-semanas

sp-Espécie

spp-Espécies

UTI-unidade de terapia intensiva

\section{Símbolos}

$$
\begin{aligned}
& >\text { - maior que } \\
& <- \text { menor que } \\
& \geq \text {-maior ou igual } \\
& \leq \text {-menor ou igual } \\
& \% \text {-por cento } \\
& \circledR \text {-Marca registrada }
\end{aligned}
$$


Unidades de medida

g-gramas

g/dl- gramas por decilitro

$\mathrm{mm}^{3}$-milímetros cúbicos 


\section{LISTA DE TABELAS}

Tabela 1 - Estatística descritiva das variáveis quantitativas idade e peso....19

Tabela 2 - Distribuição amostral segundo o peso do nascimento.................19

Tabela 3 - Distribuição amostral segundo o sexo..................................19

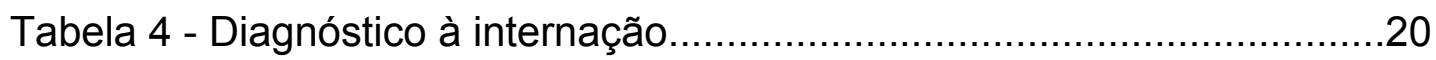

Tabela 5 - Distribuição amostral segundo fatores de risco.......................20

Tabela 6 - Distribuição amostral segundo os sinais e sintomas..................21

Tabela 7 - Local de isolamento de Candida spp em culturas......................21

Tabela 8 - Espécies de Candida identificadas nas culturas........................22

Tabela 9 - Estatística descritiva da duração do uso de antibióticos..............24

Tabela 10 - Complicações da candidíase sistêmica................................24

Tabela 11- Distribuição amostral segundo o tratamento...........................25

Tabela 12 - Resultado do hemograma............................................26

Tabela 13 - Associação entre plaquetopenia e óbito.................................26

Tabela 14 - Evolução clínica................................................................26

Tabela 15 - Comparação entre óbito e espécie de Candida.......................27

Tabela 16 - Comparação entre óbito e peso ao nascimento < 1500g..........27

Tabela 17- Comparação entre óbito e peso ao nascimento < $2500 \mathrm{~g} . . . \ldots \ldots . . .28$

Tabela 18 - Comparação entre fatores de risco e evolução clínica...............28

Tabela 19 - Comparação entre sexo e evolução clínica................................29

Tabela 20 - Comparação entre óbito e tratamento..................................29

Tabela 21 - Comparação entre óbito e tipo de preparado lipídico.................29 
Tabela 22 - Comparação entre óbito e complicações...............................30

Tabela 23 - Comparação entre espécie de Candida e complicações............30

Tabela 24 - Distribuição da freqüência de candidíase sistêmica..................31 


\section{LISTA DE GRÁFICOS}

Gráfico1: Distribuição de freqüência segundo a espécie de Candida..........22

Gráfico 2: Distribuição da freqüência do uso de antibióticos.......................23

Gráfico 3: Distribuição de freqüência segundo complicações......................25

Gráfico 4: Distribuição das espécies de Candida..................................... 31 


\section{RESUMO}

Pedroso, C.P.A. Aspectos clínicos e terapêuticos da Candidíase sistêmica em UTI neonatal: estudo de 60 casos. São Paulo, 2005. 86p. Dissertação (mestrado). Faculdade de Medicina da Univervidade de São Paulo.

INTRODUÇÃO: A candidíase sistêmica neonatal tem sido diagnosticada com freqüência crescente, devido ao aumento do número de recémnascidos de muito baixo peso e utilização de recursos tecnológicos cada vez mais sofisticados no seu tratamento. OBJETIVOS: Geral; descrever os aspectos clínicos, etiológicos e terapêuticos da candidíase sistêmica em recém-nascidos internados em Unidade de Terapia Intensiva neonatal. Específicos; descrever os sinais e sintomas e as complicações apresentadas em recém-nascidos portadores de candidíase sistêmica e as espécies de Candida; comparar a evolução clínica dos recém-nascidos considerando-se o tipo de preparado de anfotericina B utilizado no tratamento (convencional ou formulação lipídica). CASUísTICA E MÉTODOS: Estudo observacional, em 60 recém-nascidos com candidíase sistêmica internados na Unidade de Cuidados Intensivos Neonatal do Instituto da Criança do Hospital das Clínicas da Faculdade de Medicina da Universidade de São Paulo, no período de 1994 a 2003. Foi preenchido protocolo com os antecedentes perinatais, dados clínicos e exames laboratoriais e analisadas as variáveis 
categóricas e qualitativas. RESULTADOS: A freqüência anual de candidíase sistêmica no período do estudo foi, respectivamente: 1\%(1994); 0,8\%(1995);1,1\%(1996);2,2\%(1997);1,4\%(1998);2,4\%(1999);1\%(2000);1,7\% (2001);5,1\%(2002);2,1\%(2003). Houve predomínio de recém-nascidos prétermo, do sexo masculino. O baixo peso ao nascimento ocorreu em $63,3 \%$ dos recém-nascidos, sendo 50\% com peso < 1500g, e, destes 23,3\% com peso < 1000g. Fatores de risco: uso prévio de antibióticos $(96,6 \%)$, catéter venoso central (95\%), intubação traqueal (91,7\%), nutrição parenteral (90\%), prematuridade (65\%). Sinais e sintomas: alterações respiratórias, anormalidades da temperatura, letargia, hepatomegalia e esplenomegalia. Espécies de Candida: C. albicans (83,3\%), C. tropicalis (6,7\%), C.parapsilosis (5\%) e C.glabrata $(1,7 \%)$. Complicações: infecção do trato urinário $(66,7 \%)$, pneumonia $(56,7 \%)$, meningite $(13,3 \%)$, endocardite $(13,3 \%)$, trombose venosa profunda $(10 \%)$, endoftalmite $(6,7 \%)$, abscesso renal $(1,7 \%)$. O tratamento foi realizado com formulação lipídica de Anfotericina $B(70 \%)$, Anfotericina convencional (25\%) e associação de antifúngicos (5\%). A mortalidade foi de $33,3 \%$ estando associada à pneumonia $(p<0,043)$ e plaquetopenia $(p<0,0142)$. CONCLUSÃO: A freqüência global de candidíase foi de 1,8\%, sendo superior às taxas observadas recentemente por outros autores. Os sinais e sintomas mais freqüentes foram alterações respiratórias. A Candida albicans foi identificada na maioria dos casos, seguida de $C$. tropicalis, $C$. parapsilosis e $C$. glabrata,concordando com estudos da literatura. A freqüência de complicações foi levada, com mortalidade de 33,3\%, a qual foi 
significativamente associada à presença de pneumonia e plaquetopenia. A sobrevida foi significativamente maior no grupo de crianças tratadas com formulação lipídica de Anfotericina B. Os resultados obtidos evidenciam a importância da investigação sistemática de complicações multissistêmicas em todos os RN com hemocultura ou cultura de material estéril positiva para Candida sp. 


\section{SUMMARY}

Pedroso, C.P.A. Clinical and therapeutics aspects of systemic candidiasis in neonatal NICU: study of 60 cases. São Paulo, 2005.86p.

Dissertação (Mestrado) - Faculdade de Medicina da Universidade de São Paulo.

INTRODUCTION: Neonatal systemic candidiasis has been diagnosed with increasingly frequency, due to the increased number of very-low birthweight newborns and the use of more sophisticated technological resources in their treatment. OBJECTIVES: General: to describe the clinical, etiological and therapeutic aspects of systemic candidiasis in newborns admitted at the Neonatal Intensive Care Unit (NICU). Specific: to describe the signs and symptoms and the complications presented by newborns with systemic candidiasis and the Candida species; to compare the clinical evolution of the newborns considering the type of Amphotericin B preparation used in the treatment (conventional or lipid formulation). PATIENTS AND METHODS: Observational study, carried out in 60 newborns with systemic candidiasis admitted at the NICU of Instituto da Criança of Hospital das Clinicas of the University of São Paulo School of Medicine, from 1994 to 2003. A protocol that included perinatal antecedents, clinical data and laboratory assessment was filled out, and the category and qualitative variables were analyzed. 
RESULTS: The annual frequencies of systemic candidiasis during the study period were, respectively: $1 \%$ (1994); $0.8 \%$ (1995); $1.1 \%$ (1996); $2.2 \%$ (1997); 1.4\% (1998); 2.4\% (1999); 1.0\% (2000); 1.7\%(2001); 5.1\% (2002) and $2.1 \%(2003)$. There was a predominance of male preterm newborns. Low birthweight occurred in $63.3 \%$ of the newborns, with $50 \%$ of them weighing $<$ $1,500 \mathrm{~g}$ and among those, $23.3 \%$ weighed $<1,000 \mathrm{~g}$. Risk factors: previous antibiotic treatment $(96.6 \%)$, central venous catheter $(95 \%)$, tracheal intubation (91.7\%), parenteral nutrition (90\%), and prematurity (65\%). Signs and symptoms: respiratory alterations, temperature abnormalities, lethargy, hepatomegaly and splenomegaly. Candida species: C. albicans (83.3\%), C. tropicalis (6.7\%), C. parapsilosis (5\%) and C. glabrata (1.7\%). Complications: urinary tract infection (66.7\%), pneumonia (56.7\%), meningitis (13.3\%), endocarditis (13.3\%), deep venous thrombosis (10\%), endoftalmitis (6.7\%), renal abscess (1.7\%). Treatment was carried out with a lipid formulation of Amphotericin B (70\%), conventional Amphotericin (25\%) and an association of antifungal drugs (5\%). Mortality was $33.3 \%$, being associated to pneumonia $(p<0.043)$ and thrombocytopenia $(p<0.0142)$. CONCLUSION: The global frequency of candidiasis was $1.8 \%$, being higher than those observed recently by other authors. The most frequently observed signs and symptoms were respiratory alterations. Candida albicans was identified in most cases, followed by C. tropicalis, C. parapsilosis and C. glabrata, which is agreement with literature data. The complication rate was elevated, 33.3\%, and it was significantly associated to the presence of pneumonia and thrombocytopenia. The survival rate was significantly higher in the group of 
children treated with a lipid formulation of Amphotericin B. The results obtained show the importance of the systematic investigation of multisystemic complications in all newborns with a blood or sterile material culture positive for Candida sp. 
Introdução 
A candidíase sistêmica tem sido diagnosticada com freqüência crescente em Unidades de Terapia Intensiva (UTI) neonatais, devido ao aumento do número de recém-nascidos de muito baixo peso e utilização de recursos tecnológicos cada vez mais sofisticados no tratamento dessas crianças. Yamamura et al. (1999) em 14 centros do Canadá, no período de 1992 a 1994 descreveram 415 casos de candidemia, sendo $48(11,6 \%)$ em crianças, das quais 3,8\% eram recém-nascidos pré-termo. Karlowicz et al. 2000a, em 536 recém nascidos com sepse, diagnosticaram Candida sp em 18\% dos neonatos. Sax et al. (2001) em 1928 pacientes hospitalizados, incluindo adultos, crianças maiores e neonatos, relataram 142 casos de sepse, sendo os principais agentes etiológicos a Escherichia coli (19\%), o Staphylococcus aureus (12\%) e a Candida albicans (5,5\%). Lyytikäinen et al. (2002) em estudo de 1 ano com adultos, crianças e neonatos detectaram 1621 casos de infecção, sendo 65\% causadas por bactérias gram-positivas, 31\% por gram-negativas e $4 \%$ por fungos. Hajjeh et al. (2004) relataram aumento da infecção por Candida spp em Baltimore entre os anos de 1998 a 2000. Os autores detectaram 1143 casos, entre os quais $64(5,6 \%)$ ocorreram em lactentes com idade inferior a três meses, sendo 74\% neonatos. 
A mortalidade da sepse por Candida spp é elevada, variando entre 25 a $54 \%$ dos casos, podendo atingir $70 \%$ em recém-nascidos de muito baixo peso (Reyes et al. 1996) e situando-se entre 20 a $40 \%$ quando considerados os óbitos ocorridos até 30 dias de vida (Kaufman et al. 2001, Benjamin et al. 2003a).

Entre os principais fatores de risco destacam-se o uso de antibióticos de amplo espectro, como as cefalosporinas de terceira geração e o carbapenen, especialmente quando são administrados dois ou mais antibióticos (Weese 1987; Faix 1989; Ng 1994), idade gestacional inferior a 28 semanas (Benjamin et al. 2003c), ventilação mecânica, catéter venoso central, nutrição parenteral, uso de intralípides por tempo superior a sete dias, uso de medicamentos como os antagonistas da histamina (Saiman et al. 2000; Kaufman 2004), teofilina (Mehta et al. 2002), corticosteróides, vasopressores, presença de malformação congênita do trato gastrointestinal e realização de procedimento cirúrgico (Moise et al. 1986; Weese 1987; Butler 1988; Faix et al. 1989; Baley 1991; Dyke 1993; Flanagam 1998; Kossoff et al. 1998; Miller 2001; Paganini et al. 2002).

Darmstadt et al. (2000) chamaram a atenção para o maior risco de sepse por Candida spp entre recém-nascidos com candidíase cutânea congênita e peso inferior a 1000 g. Campbell et al. (2000) observaram associação entre o uso de óleos tópicos na pele de recém- 
nascidos pré-termo extremos e aumento da incidência de candidíase sistêmica.

Alguns autores relataram a importância da hiper-alimentação como fator de risco para infecção fúngica. Chowdhary et al. (2003) em 16 neonatos com candidemia, verificaram que todos os pacientes tinham recebido hiper-alimentação e pelo menos um esquema de tratamento com antibióticos.

Chiang et al. (2000) relataram um neonato pré-termo com deficiência de mieloperoxidase e candidíase sistêmica destacando a associação entre estas duas patologias.

A Candida albicans é responsável por 80 a 90\% das infecções fúngicas em humanos, estimando-se que ocorra em $75 \%$ dos casos em neonatos. Estudos destacam a importância clínica e epidemiológica de outras espécies como C. tropicalis, Candida parapsilosis, Candida lusitaniae (Butler 1988; Kao et al. 1999; Gagneur et al. 2001; Kremery et al. 2002) e Candida dubliniensis (Kim et al. 2003). No Brasil, em adultos e crianças, Colombo et al. (1999), mostraram um aumento na incidência de Candida não albicans, presente em $63 \%$ dos casos de infecção fúngica. Os autores relataram Candida albicans em 37\% dos casos, Candida parapsilosis em 25\%, Candida tropicalis em 24\%, Candida rugosa em 5\% e Candida glabrata em 4\%. Em neonatos, paralelamente ao aumento da incidência de sepse fúngica, observa-se a emergência de diferentes espécies de Candida, refletindo, 
provavelmente, uma mudança do perfil da candidíase sistêmica nas Unidades de Terapia Intensiva neonatais (Hostetter 1996; Malani et al.2000; Kremery et al. 2002; Brandt et al. 2003). Roilides et al. (2003) revisaram casos de infecção em neonatos durante 1 ano relatando 10 casos de candidemia, identificando Candida parapsilosis, Candida tropicalis e Candida glabrata sendo todos eram pacientes previamente colonizados.

As manifestações clínicas de candidíase sistêmica são variadas, com início geralmente insidioso e sinais e sintomas que podem ser confundidos com aqueles observados na infecção bacteriana (Faix 1984; Patrick 1992; Hostetter 1996; Reyes et al. 1996). A ausência de bactérias nas culturas com persistência dos sintomas, em recém-nascidos com fatores de risco, sugerem fortemente 0 diagnóstico de infecção fúngica. Os achados são inespecíficos: instabilidade térmica, hipoatividade, distensão abdominal, fezes guaiaco positivas, hiperglicemia, crises de apnéia, episódios de bradicardia, hepatomegalia, esplenomegalia, icterícia e infiltrado pulmonar no RX de tórax ( Faix 1984; Miller 2001). Klingspor et al. (1993) estudaram a freqüência de Candida $s p$ nas fezes de 119 crianças com diarréia, observando Candida spp em 32\% dos casos, Candida tropicalis em 19\% Candida albicans em 6\% e Candida parapsilosis em $3 \%$ dos casos. 
As complicações multissistêmicas da candidíase neonatal são freqüentes, devido à capacidade do fungo promover invasão tecidual em diferentes órgãos, como pulmão, fígado, baço, rim, coração, retina, cérebro, e articulações (Perrone 1970; Baley 1981; Faix 1992; Weintrub et al. 1994; Chapman et al. 2000; Ko et al. 2000; Warady et al.2000; Noyola et al., 2001; Robertson et al.2003; Chapman et al. 2003; Wimalendra et al.2004 ). Benjamin et al. (2003b) em uma meta-análise, relataram as seguintes taxas de prevalência média: 15\% de meningite, $4 \%$ de abscesso cerebral e ventriculite, $5 \%$ de endocardite, $3 \%$ de endoftalmite, $5 \%$ de abscesso renal e $1 \%$ de abscesso hepático ou esplênico. Doerr et al. (1994) descreveram três casos de abscesso hepático em neonatos, sendo dois causados por fungos (Malassezia furfur e Candida parapsilosis, respectivamente). Strong et al. (1995) entre sete crianças com artrite séptica, relataram um paciente com cultura de líquido articular positiva para Candida albicans e comprometimento de 18 articulações.

A infecção do sistema nervoso central por Candida spp pode causar diferentes tipos de lesões, como vasculite, meningite, ventriculite, cerebelite, infarto, e abscesso cerebral. O abscesso intraventricular é uma complicação rara. Winters et al. (1995) descreveram um caso incomum de abscesso intra-ventricular em um neonato com meningite por Candida albicans que faleceu após 11 dias, apesar do tratamento apropriado. 
O diagnóstico definitivo de candidíase sistêmica baseia-se no isolamento do fungo em cultura de sangue ou material de biópsia. A pesquisa direta na urina, exame microscópico direto de raspado de lesão cutânea, fluidos aspirados ou biópsias, são exames mais rápidos, que podem auxiliar na indicação do tratamento, enquanto se aguarda o resultado das culturas. Os exames sorológicos, para detecção de antígenos fúngicos e o uso de reação em cadeia da polimerase ainda não são disponíveis para o uso na prática diária (Walsh et al. 1995; Sigmundsdóttir et al. 2000; Tirodker et al. 2003). Devido à inespecificidade dos sinais e sintomas clínicos, um dos aspectos mais importantes na elucidação diagnóstica da candidíase disseminada é o alto índice de suspeita clínica, especialmente em recém-nascidos de muito baixo com fatores de risco para infecção fúngica. Chapman et al. (2000) realizaram estudo para verificar a persistência de positividade das culturas em neonatos com candidíase sistêmica entre 1981 a 1999. Entre os 96 neonatos incluídos no estudo, 58 apresentaram infecção persistente, com culturas de sangue e/ou líquor positivas no sétimo, $14^{\circ}$ e $21^{\circ}$ dias. Os autores observaram que a freqüência de complicações, como uropatia, endocardite, abscesso, ventriculite, e dermatite invasiva e óbito foram significativamente maiores entre os recém-nascidos com culturas persistentemente positivas.

O tratamento de escolha da candidíase sistêmica em recémnascidos é a anfotericina B, macrolídeo polieno que age ligando o 
ergosterol à membrana celular fúngica, provocando sua lise, com extravasamento do conteúdo citoplasmático e morte celular (Anker et al. 1995; Bennet 2001; Frattanelli et al. 2004). Seus efeitos colaterais são freqüentes e potencialmente graves: febre, broncoespasmo, nefrotoxicidade, necrose hepática e supressão medular (Glick et al.1993; Reyes et al. 1996; Scarcella et al. 1998; Krebs et al. 1999a). O tratamento deve ser mantido até atingir a dose acumulada de 25-30 $\mathrm{mg} / \mathrm{Kg}$ (Miller et al. 2001) ou 50mg/kg na presença de complicações graves, como endocardite ou meningite (Sánchez et al. 1991). Com o advento dos preparados lipídicos de anfotericina $B$, tornou-se possível administrar altas doses da droga, com menor toxicidade, melhorando assim sua eficácia clínica (Krebs et al. 1999a).

Pelo exposto, podemos observar que a candidíase sistêmica neonatal é uma doença grave, cujos aspectos clínicos e terapêuticos ainda não são completamente conhecidos. A incidência exata de complicações como meningite, endocardite e endoftalmite em nosso meio é desconhecida e, até o momento, há poucas publicações analisando o tratamento com preparados lipídicos de anfotericina B. Nossa hipótese é que o maior conhecimento do comportamento da candidíase sistêmica neonatal ao longo de uma década em nosso meio possa auxiliar na prevenção e tratamento desta grave doença. 
Objetivos 


\section{OBJETIVO GERAL:}

Descrever os aspectos clínicos, etiológicos e terapêuticos da candidíase sistêmica em recém-nascidos internados em Unidade de Terapia Intensiva neonatal.

\section{OBJETIVOS ESPECÍFICOS:}

- Descrever os sinais e sintomas e as complicações apresentadas em recém-nascidos portadores de candidíase sistêmica.

- Descrever as espécies de Candida identificadas nesses recémnascidos.

- Comparar a evolução clínica dos recém-nascidos tratados considerando-se o tipo de preparado de anfotericina B utilizado no tratamento (convencional ou formulação lipídica). 
Casuística e Métodos 
Foi realizado um estudo observacional em 60 recém-nascidos com candidíase sistêmica internados na UCINE (Unidade de Cuidados Intensivos Neonatal) do Instituto da Criança do Hospital das Clínicas da Faculdade de Medicina da Universidade de São Paulo, no período de 1994 a 2003.

\section{Definição de candidíase sistêmica}

Candidíase sistêmica foi definida como a presença de sinais e sintomas de infecção e candidemia ou isolamento de Candida spp. em cultura de material normalmente estéril, obtido de locais fechados (líquor, urina obtida por punção supra-púbica, líquido articular e líquido peritonial).

\section{Definição de candidemia}

Candidemia foi definida como a presença de pelo menos uma cultura de sangue positiva para Candida spp. 


\section{Pneumonia}

Para o diagnóstico de pneumonia por Candida foram utilizados os seguintes critérios: presença de sinais e sintomas respiratórios e piora radiológica pulmonar em neonatos com hemocultura ou cultura de material normalmente estéril positiva para Candida spp.

\section{Critérios de inclusão:}

Foram incluídos no estudo todos os recém-nascidos que apresentaram candidíase sistêmica, admitidos durante o período de estudo.

\section{Critérios de exclusão:}

Foram excluídos do estudo os recém-nascidos com quadro clínico compatível com infecção fúngica, porém sem identificação de Candida spp. nas culturas ou com isolamento apenas em ponta de catéter. 


\section{MÉTODOS}

Foi realizada análise de prontuário de 60 recém-nascidos com candidíase sistêmica, admitidos no período de janeiro de 1994 a dezembro de 2003.

Para cada paciente foi preenchido um protocolo com os dados clínicos e resultados dos exames laboratoriais. As variáveis analisadas foram: peso de nascimento, idade gestacional, idade à internação, tipo de parto, sinais e sintomas, tipo de tratamento (anfotericina B convencional e formulação lipídica de anfotericina), complicações da candidíase sistêmica, espécies de Candida identificadas nas culturas, resultado do exame do líquido cefalorraqueano, oftalmoscopia indireta, ultra-sonografia de abdome e de crânio, RX de tórax e ecocardiograma. Foram também considerados a ocorrência de plaquetopenia (número de plaquetas $<100000 / \mathrm{mm}^{3}$ ), plaquetose (número de plaquetas $>400000 / \mathrm{mm}^{3}$ ), leucocitose (número de

leucócitos $>21000 / \mathrm{mm}^{3}$ ), leucopenia (número de leucócitos $<5000 / \mathrm{mm}^{3}$, eosinofilia (número de eosinófilos $\geq 10 / \mathrm{mm}^{3}$ ). 


\section{Análise estatística}

Foi realizada a estatística descritiva das características clínicas e espécies de Candida identificadas e do tratamento utilizado. Com o objetivo de caracterizar a amostra estudada, foram apresentados as freqüências relativas (percentuais) e absolutas $(\mathrm{N})$ das classes de cada variável qualitativa( variáveis categóricas). Para as variáveis quantitativas(contínuas) foram utilizadas médias e medianas para resumir as informações, e desviospadrão, mínimo e máximo para indicar a variabilidade dos dados.

Para comparar as distribuições de freqüência das variáveis qualitativas foi utilizado o teste qui-quadrado, que se baseia nas diferenças entre valores observados e esperados, avaliando se as proporções em cada grupo podem ser consideradas semelhantes ou não. $O$ teste exato de Fisher foi utilizado nas situações onde os valores esperados foram inferiores a 5 . Foram considerados significantes os valores de $p$ menores do que 0,05 .

\section{Aprovação pela Comissão de Pesquisa e Ética}

O projeto de pesquisa foi aprovado pela Comissão de Ética para Análise de Projetos de Pesquisa (CAPPesq) do Hospital das Clínicas da Faculdade de Medicina da Universidade de São Paulo, em 10 de abril de 2002. (protocolo 383/01). 
Resultados 
No período de janeiro de 1994 a dezembro de 2003 (10 anos) foram admitidos 3219 recém-nascidos na UCINE, sendo 60 (1,8\%) com diagnóstico de candidíase sistêmica, que constituíram a casuística do presente estudo. A freqüência anual da doença variou de 0,8 \%, em 1995, a 5,1\% no ano de 2002. A distribuição dos recém-nascidos segundo a idade, peso de nascimento, sexo, idade gestacional e duração da internação estão apresentados nas tabelas 1, 2, 3 . 
Tabelas 
Tabela 1 - Estatística descritiva das variáveis quantitativas idade e peso de nascimento em 60 recém nascidos com candidíase sistêmica internados em UTI neonatal -1994 a 2003

\begin{tabular}{ccccccc}
\hline \hline Variáveis & $\mathbf{N}$ & Média & Mediana & $\begin{array}{c}\text { Desvio } \\
\text { Padrão }\end{array}$ & Mínimo & Máximo \\
\hline \hline Idade à internação (d) & 60 & 7,42 & 1,5 & 12,38 & 1 & 66 \\
Idade do início da doença(d) & 60 & 31,57 & 24,5 & 21,28 & 8 & 120 \\
Idade gestacional (sem.) & 60 & 33,52 & 32 & 4,71 & 24 & 40 \\
Peso de nascimento (g) & 60 & 1960 & 1512,5 & 1118,45 & 690 & 5000 \\
Tempo de internação (d) & 60 & 67,92 & 60 & 37,38 & 16 & 210 \\
\hline \hline
\end{tabular}

Tabela 2- Distribuição amostral segundo o peso do nascimento categorizado em 60 recém-nascidos com candidíase sistêmica internados em UTI neonatal -1994 a 2003

\begin{tabular}{ccc}
\hline \hline Peso do nascimento & $\mathbf{N}$ & $\%$ \\
\hline$<1000 \mathrm{~g}$ & 14 & 23,3 \\
$1000-1499 \mathrm{~g}$ & 16 & 26,7 \\
$1500-2499 \mathrm{~g}$ & 8 & 13,3 \\
$\geq 2500 \mathrm{~g}$ & 22 & 36,7 \\
TOTAL & 60 & 100,0 \\
\hline \hline
\end{tabular}

Tabela 3- Distribuição amostral segundo o sexo em 60 recém-nascidos com candidíase sistêmica internados em UTI neonatal -1994 a 2003

\begin{tabular}{ccc}
\hline \hline Sexo & $\mathbf{N}$ & \% \\
\hline Masculino & 39 & 65 \\
Feminino & 21 & 35 \\
TOTAL & 60 & 100 \\
\hline \hline
\end{tabular}


Tabela 4- Diagnóstico à internação em 60 recém-nascidos com candidíase sistêmica internados em UTI neonatal -1994 a 2003

\begin{tabular}{ccc}
\hline \hline Diagnóstico & $\mathbf{n}$ & $\%$ \\
\hline Prematuridade & 39 & 65 \\
Baixo peso ao nascimento & 38 & 63 \\
Doença das membranas hialinas & 25 & 42 \\
Asfixia perinatal & 20 & 33 \\
Sepse & 20 & 33 \\
Restrição de crescimento intra-uterino & 12 & 20 \\
Pneumonia & 11 & 18 \\
Hemorragia intracraniana & 9 & 15 \\
Enterocolite Necrosante & 7 & 12 \\
Persistência do canal arterial & 6 & 10 \\
Malformação do aparelho digestivo & 6 & 10 \\
Pneumotórax & 6 & 10 \\
Distúrbio do metabolismo da glicose & 6 & 10 \\
Meningomielocele & 4 & 7 \\
Malformação do sistema urinário & 3 & 5 \\
Outros & 14 & 23 \\
\hline \hline
\end{tabular}

Tabela 5- Distribuição amostral segundo fatores de risco em 60 recémnascidos com candidíase sistêmica internados em UTI neonatal 1994 a 2003

\begin{tabular}{ccc}
\hline \hline Fatores de Risco & N & $\%$ \\
\hline Antibióticos & 58 & 96,6 \\
Catéter venoso central & 57 & 95,0 \\
Intubação traqueal & 55 & 91,7 \\
Nutrição parenteral & 54 & 90,0 \\
Prematuridade & 39 & 65,0 \\
Sexo masculino & 39 & 65,0 \\
Baixo peso ao nascimento & 38 & 63,3 \\
Teofilina & 24 & 40,0 \\
Cirurgia do aparelho digestivo & 14 & 23,3 \\
Restrição do crescimento intra-uterino & 12 & 20,0 \\
Corticóide & 10 & 16,7 \\
Dreno torácico & 10 & 16,7 \\
Malformação & 4 & 6,7 \\
Gastrostomia & 1 & 1,7 \\
Quimioterápicos & 1 & 1,7 \\
Colostomia & 1 & 1,7 \\
Catéter de diálise & 1 & 1,7 \\
\hline \hline
\end{tabular}


Tabela 6- Distribuição amostral segundo os sinais e sintomas em 60 recémnascidos com candidíase sistêmica internados em UTI neonatal 1994 a 2003

\begin{tabular}{ccc}
\hline \hline Sinais e Sintomas & N & $\%$ \\
\hline \hline Estertores pulmonares & 38 & 63,3 \\
Piora radiológica pulmonar & 36 & 60,0 \\
Taquipnéia & 24 & 40,0 \\
Febre & 38 & 63,3 \\
Hipotermia & 33 & 55,0 \\
Letargia & 33 & 55,0 \\
Hepatomegalia & 31 & 51,7 \\
Sopro cardíaco & 27 & 45,0 \\
Distensão abdominal & 24 & 40,0 \\
Esplenomegalia & 20 & 33,3 \\
Apnéia & 17 & 28,3 \\
Bradicardia & 14 & 23,3 \\
Icterícia & 14 & 23,3 \\
Monilíase cutânea & 12 & 20,0 \\
Recusa alimentar & 10 & 16,7 \\
Vômitos & 9 & 15,0 \\
Convulsões & 8 & 13,3 \\
Petéquias & 8 & 13,3 \\
Sangramento digestivo & 8 & 13,3 \\
Irritabilidade & 7 & 11,7 \\
\hline \hline
\end{tabular}

Tabela 7- Local de isolamento de Candida spp em culturas em 60 recémnascidos com candidíase sistêmica internados em UTI neonatal 1994 a 2003

\begin{tabular}{ccc}
\hline \hline Local de isolamento* & $\mathbf{n}$ & $\%$ \\
\hline Sangue & 17 & 28,3 \\
Urina & 16 & 26,6 \\
Sangue e urina & 16 & 26,6 \\
Sangue ,urina e catéter & 3 & 5,0 \\
Sangue e catéter & 2 & 3,3 \\
Sangue ,urina e líquor & 1 & 1,7 \\
Sangue, urina ,catéter,líquido peritonial & 1 & 1,7 \\
Urina e catéter & 1 & 1,7 \\
Urina e líquor & 1 & 1,7 \\
Líquido peritonial & 1 & 1,7 \\
Urina,líquor e líquido peritonial & 1 & 1,7 \\
\hline \hline TOTAL & 60 & 100 \\
\hline \hline
\end{tabular}

hemocultura $=40(66,7 \%)$; urocultura $=40(66,7 \%)$;

ponta de catéter $=3(5,0 \%)$; líquor $=3(5,0 \%) ;$ líquido peritonial $=3(5,0 \%)$ 
Tabela 8 - Espécies de Candida identificadas nas culturas em 60 recémnascidos com candidíase sistêmica internados em UTI neonatal -1994 a 2003

\begin{tabular}{ccc}
\hline \hline Espécies de Candida & $\mathbf{N}$ & $\%$ \\
\hline Candida albicans & 50 & 83,3 \\
Candida tropicalis & 4 & 6,7 \\
Candida parapsilosis & 3 & 5,0 \\
Candida glabrata & 1 & 1,7 \\
Candida sp & 2 & 3,3 \\
\hline TOTAL & 60 & 100 \\
\hline \hline
\end{tabular}

Gráfico 1 - Distribuição de freqüência segundo a espécie de Candida em 60 recém-nascidos com candidíase sistêmica internados em UTI neonatal - 1994 a 2003

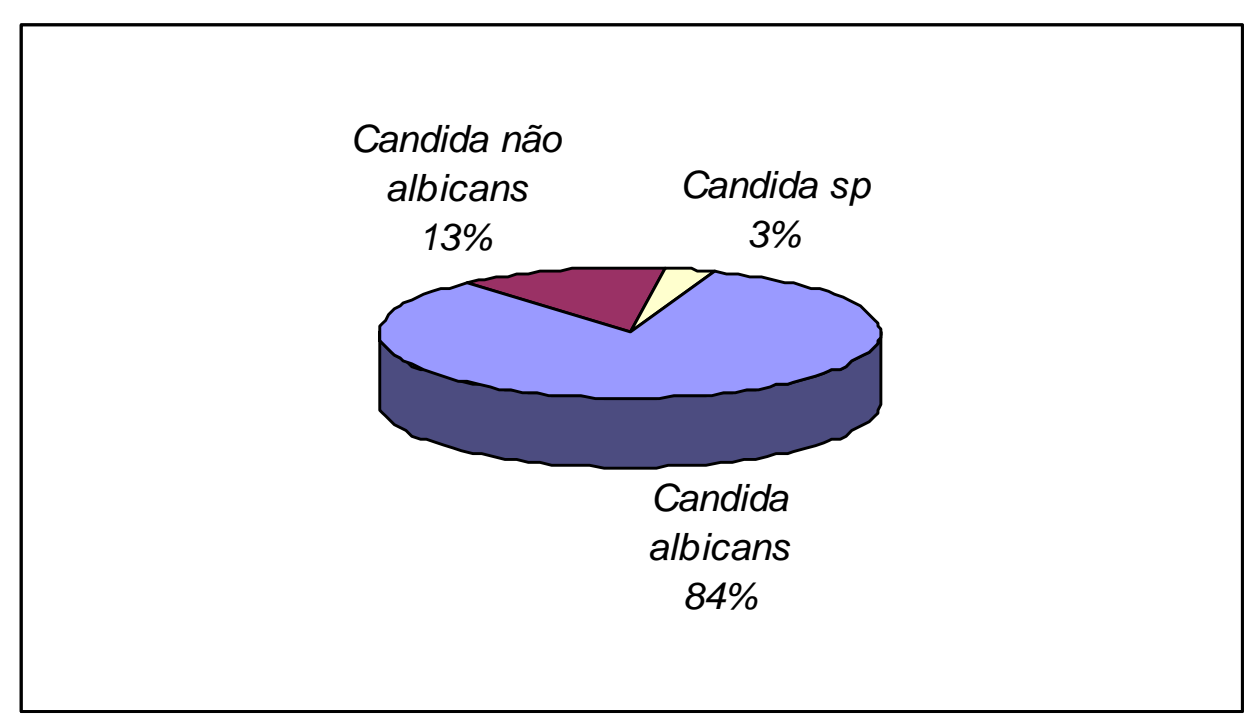


Gráfico 2 - Distribuição da freqüência do uso de antibióticos em 60 recémnascidos com candidíase sistêmica internados em UTI neonatal1994 a 2003

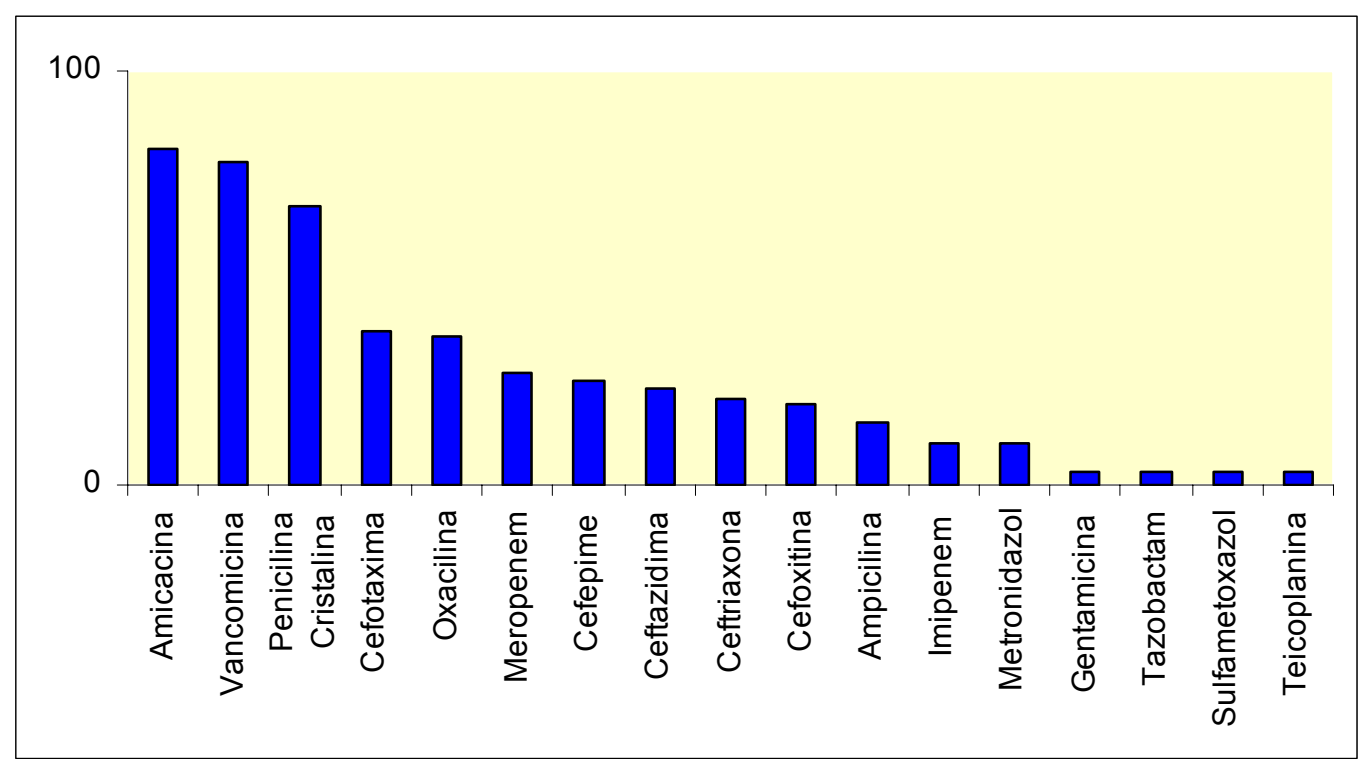


Tabela 9- Estatística descritiva da duração do uso de antibióticos em 60 recém- nascidos com candidíase sistêmica internados em UTI neonatal-1994 a 2003

\begin{tabular}{|c|c|c|c|c|c|c|}
\hline \multirow{2}{*}{ Antibiótico } & \multicolumn{6}{|c|}{ Duração (d) } \\
\hline & $N$ & Média & Mediana & $\begin{array}{l}\text { Desvio } \\
\text { Padrão }\end{array}$ & Mínimo & Máximo \\
\hline Amicacina & 49 & 9,18 & 8 & 5,53 & 2 & 31 \\
\hline Vancomicina & 47 & 15,21 & 14 & 13,24 & 1 & 87 \\
\hline Penicilina cristalina & 40 & 8,08 & 8 & 3,53 & 2 & 14 \\
\hline Cefotaxima & 22 & 9,59 & 8,5 & 5,94 & 1 & 21 \\
\hline Oxacilina & 21 & 7,05 & 4 & 6,84 & 1 & 29 \\
\hline Meropenem & 16 & 17,69 & 13,5 & 18,97 & 3 & 83 \\
\hline Cefepime & 15 & 10,27 & 10 & 7,17 & 1 & 22 \\
\hline Ceftazidima & 14 & 12,5 & 14 & 8,37 & 2 & 34 \\
\hline Ceftriaxona & 13 & 7,38 & 7 & 4,61 & 1 & 14 \\
\hline Mefoxim & 12 & 8,17 & 9,5 & 4,32 & 1 & 14 \\
\hline Ampicilina & 9 & 10 & 8 & 6,56 & 2 & 21 \\
\hline Imipenem & 6 & 14,67 & 14,5 & 4,41 & 9 & 21 \\
\hline Metronidazol & 6 & 10,67 & 11 & 5,57 & 3 & 18 \\
\hline Tazobactam & 1 & 19 & 19 & - & 19 & 19 \\
\hline Sulfametoxazol-trimetoprim & 1 & 4 & 4 & - & 4 & 4 \\
\hline Gentamicina & 1 & 8 & 8 & - & 8 & 8 \\
\hline Teicoplanina & 1 & 6 & 6 & - & 6 & 6 \\
\hline
\end{tabular}

Tabela 10- Complicações da candidíase sistêmica em 60 recém-nascidos internados em UTI neonatal -1994 a 2003

\begin{tabular}{ccc}
\hline \hline Complicações & N & $\%$ \\
\hline Infecção urinária & 40 & 66,7 \\
Pneumonia & 34 & 56,7 \\
Meningite & 8 & 13,3 \\
Endocardite & 8 & 13,3 \\
Trombose veia cava superior & 6 & 10,0 \\
Endoftalmite & 4 & 6,7 \\
Abscesso renal & 1 & 1,7 \\
\hline \hline
\end{tabular}


Gráfico 3-Distribuição de freqüência segundo complicações em 60 recémnascidos com candidíase sistêmica internados em UTI neonatal 1994 a 2003



Tabela 11-Distribuição amostral segundo o tratamento em 60 recémnascidos com candidíase sistêmica internados em UTI neonatal 1994 a 2003

\begin{tabular}{ccc}
\hline \hline Tratamento & N & $\%$ \\
\hline Anfotericina convencional & 15 & 25 \\
Anfotericina formulação lipídica & 42 & 70 \\
Anfotericina convencional e lipídica & 3 & 5 \\
TOTAL & 60 & 100 \\
\hline \hline
\end{tabular}


Tabela 12- Resultado do hemograma em 60 neonatos com candidíase sistêmica -1994 a 2003

\begin{tabular}{ccc}
\hline \hline Resultado do hemograma $^{*}$ & $\mathbf{n}$ & $\%$ \\
\hline Plaquetopenia & 28 & 46,7 \\
Eosinofilia & 10 & 16,7 \\
Normal & 9 & 15,0 \\
Leucocitose & 6 & 10,0 \\
Leucopenia & 5 & 8,3 \\
Plaquetose & 2 & 3,3 \\
\hline TOTAL & 60 & 100,0 \\
\hline \hline
\end{tabular}

* Foram observadas alterações em 51 (85\%) neonatos

*Fonte: Nathan and Oskis. Hematology of Infancy and childhood. 1998

Tabela 13-Associação entre plaquetopenia e óbito em 60 recém-nascidos com candidíase sistêmica internados em UTI neonatal -1994 a 2003

\begin{tabular}{ccccc}
\hline \hline Número de plaquetas & $\begin{array}{c}\text { Óbito } \\
\mathbf{N}(\%)\end{array}$ & $\begin{array}{c}\text { Sobrevida } \\
\mathbf{N}(\%)\end{array}$ & $\begin{array}{c}\text { TOTAL } \\
\mathbf{N}(\%)\end{array}$ & $\mathrm{p}$ \\
\hline Com plaquetopenia & $14(23,3)$ & $14(23,3)$ & $28(47,0)$ & $0,0142_{F}$ \\
Sem plaquetopenia & $6(10,0)$ & $26(43,0)$ & $32(53,0)$ & \\
TOTAL & $20(33,3)$ & $40(67,7)$ & $60(100,0)$ & \\
\hline \hline
\end{tabular}

Tabela 14- Evolução clínica em 60 recém-nascidos com candidíase sistêmica internados em UTI neonatal-1994 a 2003

\begin{tabular}{ccc}
\hline \hline Evolução clínica & N & $\%$ \\
\hline Alta & 40 & 66,7 \\
Óbito & 20 & 33,3 \\
TOTAL & 60 & 100,0 \\
\hline \hline
\end{tabular}




\section{ANÁLISE ESTATÍSTICA}

Análise da mortalidade relacionada à candidíase sistêmica.

Tabela 15- Comparação entre óbito e espécie de Candida em recémnascidos com candidíase sistêmica internados em UTI neonatal 1994 a 2003

\begin{tabular}{rlcccc}
\hline \hline & & Alta & Óbito & TOTAL* $^{*}$ & $\mathrm{p}$ \\
\cline { 2 - 5 } & & $\mathrm{N}(\%)$ & $\mathrm{N}(\%)$ & & \\
\hline \hline \multirow{3}{*}{ Espécie de Candida } & $\begin{array}{c}\text { Candida } \\
\text { albicans }\end{array}$ & $34((59,0)$ & $16(28,0)$ & $50(83,3)$ & \\
\cline { 2 - 5 } & $\begin{array}{c}\text { Candida não } \\
\text { albicans }\end{array}$ & $4(7,0)$ & $4(7,0)$ & $8(14,0)$ & $0,427_{\mathrm{F}}$ \\
\hline TOTAL & & $38(66,0)$ & $20(34)$ & $58(100,0)$ &
\end{tabular}

$\overline{{ }^{*} \text { Não foi identificada a espécie em dois }} \overline{\overline{c a s o s}}$

Tabela 16- Comparação entre óbito e peso ao nascimento $<1500 \mathrm{~g}$ em 60 recém-nascidos com candidíase sistêmica internados em UTI neonatal -1994 a 2003

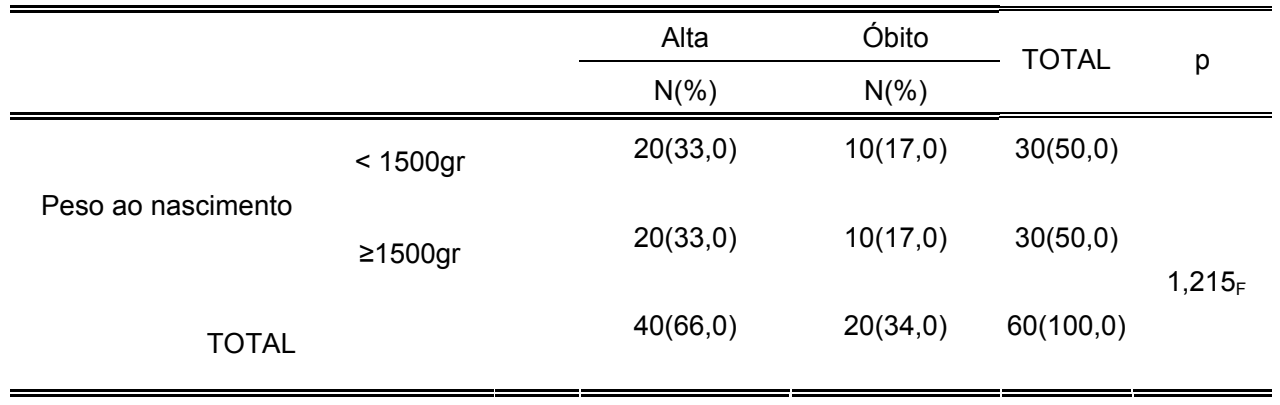


Tabela 17- Comparação entre óbito e baixo peso ao nascimento em 60 recém-nascidos com candidíase sistêmica internados em UTI neonatal -1994 a 2003

\begin{tabular}{|c|c|c|c|c|c|}
\hline & & $\begin{array}{l}\text { Alta } \\
\mathrm{N}(\%)\end{array}$ & $\begin{array}{l}\text { Óbito } \\
N(\%)\end{array}$ & TOTAL & $P$ \\
\hline \multirow{3}{*}{ Peso ao nascimento } & $<2500 \mathrm{~g}$ & $24(60,0)$ & $14(70,0)$ & 38 & \multirow{4}{*}{$0,573_{\mathrm{F}}$} \\
\hline & & & & & \\
\hline & $\geq 2500 \mathrm{~g}$ & $16(40,0)$ & $6(30,0)$ & 22 & \\
\hline TOTAL & & 40 & 20 & 60 & \\
\hline
\end{tabular}

Tabela 18-Comparação entre fatores de risco e evolução clínica em 60 recém-nascidos com candidíase sistêmica internados em UTI neonatal -1994 a 2003

\begin{tabular}{|c|c|c|c|c|}
\hline \multirow[b]{2}{*}{ Fatores de Risco } & \multirow[b]{2}{*}{$N(\%)$} & \multicolumn{2}{|c|}{ "Evolução } & \multirow[t]{2}{*}{$\overline{\bar{p}}$} \\
\hline & & $\begin{array}{c}\text { Alta }(n=40) \\
N(\%)\end{array}$ & $\begin{array}{c}\text { Óbito }(n=20) \\
N(\%)\end{array}$ & \\
\hline Antibióticos & $58(96,6)$ & $38(63,0)$ & $20(33,0)$ & 0,548 \\
\hline Catéter venoso central & $57(95,0)$ & $37(62,0)$ & $20(33,0)$ & 0,544 \\
\hline Intubação traqueal & $55(91,7)$ & $35(58,0)$ & $20(33,0)$ & 0,158 \\
\hline Nutrição parenteral & $54(90,0)$ & $36(60,0)$ & $19(32,0)$ & 0,656 \\
\hline Prematuridade & $39(65,0)$ & $25(42,0)$ & $14(23,0)$ & 0,774 \\
\hline Sexo masculino & $39(65,0)$ & $27(45,0)$ & $12(20,0)$ & 0,579 \\
\hline Baixo peso ao nascimento & $38(63,3)$ & $24(40,0)$ & $14(23,3)$ & 0,572 \\
\hline Teofilina & $24(40,0)$ & $17(28,0)$ & $7(12,0)$ & 0,780 \\
\hline Cirurgia do aparelho digestivo & $14(23,3)$ & $8(13,0)$ & $6(10,0)$ & 0,518 \\
\hline Malformação & $13(21,6)$ & $9(15,0)$ & $4(7,0)$ & 1,000 \\
\hline Restrição do crescimento intra uterino & $12(20,0)$ & $6(10,0)$ & $6(10,0)$ & 0,189 \\
\hline Corticóide & $10(16,7)$ & $7(12,0)$ & $3(5,0)$ & 1,000 \\
\hline Dreno torácico & $10(16,7)$ & $5(8,0)$ & $5(8,0)$ & 0,277 \\
\hline Gastrostomia & $1(1,7)$ & $1(1,7)$ & - & 1,000 \\
\hline Quimioterápicos & $1(1,7)$ & - & $1(1,7)$ & 0,333 \\
\hline Colostomia & $1(1,7)$ & $1(1,7)$ & - & 1,000 \\
\hline Catéter de diálise & $1(1,7)$ & $1(1,7)$ & - & 1,000 \\
\hline
\end{tabular}


Tabela 19 - Comparação entre sexo e evolução clínica em 60 recémnascidos com candidíase sistêmica internados em UTI neonatal 1994 a 2003

\begin{tabular}{ccccc}
\hline \hline Sexo & $\mathrm{N}(\%)$ & $\begin{array}{c}\text { Alta } \\
\mathrm{N}(\%)\end{array}$ & $\begin{array}{c}\text { Óbito } \\
\mathrm{N}(\%)\end{array}$ & $\mathrm{p}$ \\
\hline \hline Masculino & $39(65,0)$ & $25(42,0)$ & $14(23,0)$ & \\
Feminino & $21(35,0)$ & $15(25,0)$ & $6(10,0)$ & \multirow{2}{*}{$0,774 \mathrm{~F}$} \\
Total & $60(100,0)$ & $40(67,0)$ & $20(33,0)$ & \\
\hline \hline
\end{tabular}

Tabela 20- Comparação entre óbito e tratamento em 57 recém-nascidos com candidíase sistêmica internados em UTI neonatal -1994 a 2003

\begin{tabular}{ccccc}
\hline \hline & Alta & Óbito & & \\
\cline { 2 - 3 } Tratamento & $\mathrm{N}(\%)$ & $\mathrm{N}(\%)$ & & $\mathrm{p}$ \\
\hline \hline Anfotericina B convencional & $5(9,0)$ & $10(18,0)$ & $15(26,0)$ & \\
Anfotericina formulação lipídica & & & & $0,0031_{F}$ \\
TOTAL & $33(58,0)$ & $9(16,0)$ & $42(74,0)$ & \\
& $38(67,0)$ & $19(33,0)$ & $57(100,0)$ \\
\hline \hline
\end{tabular}

$\overline{\text { *Em } 3 \text { casos houve associação de anfotericina convencional e lipídica }} \overline{\bar{e}}$

Tabela 21- Comparação entre óbito e tipo de preparado lipídico no tratamento em 42 recém-nascidos com candidíase sistêmica internados em UTI neonatal -1994 a 2003

\begin{tabular}{|c|c|c|c|}
\hline \multirow[b]{2}{*}{ Tipo de preparado lipídico } & Alta & Óbito & \multirow{2}{*}{ TOTAL } \\
\hline & $\mathrm{N}(\%)$ & $\mathrm{N}(\%)$ & \\
\hline Anfotericina liposomal & $7(17,0)$ & $3(7,0)$ & $10(24,0)$ \\
\hline Anfotericina dispersão coloidal & $26(62,0)$ & $6(14,0)$ & $32(76,0) \quad 0,660_{F}$ \\
\hline TOTAL & $33(79,0)$ & $9(21,0)$ & $42(100,0)$ \\
\hline
\end{tabular}


Tabela 22-Comparação entre óbito e complicações em 60 recém-nascidos com candidíase sistêmica internados em UTI neonatal -1994 a 2003

\begin{tabular}{ccccc}
\hline \hline $\begin{array}{c}\text { Tipo de Complicação* } \\
(\mathrm{N}=60)\end{array}$ & Freqüência & \multicolumn{2}{c}{ Alta(n=40) } & Óbito(n=20) \\
& $\mathrm{N}(\%)$ & $\mathrm{N}(\%)$ & $\mathrm{N}(\%)$ & $\mathrm{p}$ \\
\hline \hline Infecção urinária & $40(66,7 \%)$ & $29(48,3 \%)$ & $11(18,3 \%)$ & $0,246_{\mathrm{F}}$ \\
Pneumonia & $34(56,7 \%)$ & $19(31,7 \%)$ & $15(25,0 \%)$ & $0,055_{\mathrm{F}}$ \\
Endocardite & $8(13,3 \%)$ & $5(8,3 \%)$ & $3(5,0 \%)$ & $1,000 \mathrm{~F}$ \\
Meningite & $8(13,3 \%)$ & $6(10,0 \%)$ & $2(3,3 \%)$ & $0,707_{\mathrm{F}}$ \\
Trombose venosa & $6(10,0 \%)$ & $2(3,3 \%)$ & $4(6,7 \%)$ & $0,089_{\mathrm{F}}$ \\
Endoftalmite & $4(6,7 \%)$ & $2(3,3 \%)$ & $2(3,3 \%)$ & $0,595_{\mathrm{F}}$ \\
Abscesso renal & $1(1,7 \%)$ & $0(0,0 \%)$ & $1(1,7 \%)$ & $0,333_{\mathrm{F}}$ \\
\hline \hline
\end{tabular}

*Alguns recém-nascidos apresentaram associação de duas ou mais complicações

Tabela 23- Comparação entre espécie de Candida e complicações em 60 recém- nascidos com candidíase sistêmica internados em UTI neonatal -1994 a 2003

\begin{tabular}{|c|c|c|c|c|}
\hline \multirow{2}{*}{ Complicação* } & \multicolumn{2}{|c|}{ Espécie de Candida** } & \multirow{2}{*}{ TOTAL } & \multirow[b]{2}{*}{$\mathrm{p}$} \\
\hline & $\begin{array}{c}\text { C albicans } \\
\mathrm{N}(\%)\end{array}$ & $\begin{array}{c}\text { C.não albicans } \\
\mathrm{N}(\%)\end{array}$ & & \\
\hline Infecção urinária & $33(55,0)$ & $7(11,7)$ & $40(66,7)$ & $1,000 \mathrm{~F}$ \\
\hline Pneumonia & $29(48,3)$ & $5(8,3)$ & $34(56,7)$ & $0,733_{\mathrm{F}}$ \\
\hline Meningite & $8(13,0)$ & $0(0,0)$ & $8(13,3)$ & $0,330_{F}$ \\
\hline Endocardite & $5(8,3)$ & $3(5,0)$ & $8(13,3)$ & $0,120_{\mathrm{F}}$ \\
\hline Trombose veia cava superior & $6(10,0)$ & $0(0,0)$ & $6(10,0)$ & $0,577_{\mathrm{F}}$ \\
\hline Endoftalmite & $4(6,7)$ & $0(0,0)$ & $4(6,7)$ & $1,000 \mathrm{~F}$ \\
\hline Abscesso renal & $1(1,7)$ & $0(0,0)$ & $1(1,7)$ & $1,000 \mathrm{~F}$ \\
\hline
\end{tabular}

*Alguns recém-nascidos apresentaram associação de duas ou mais complicações

** $C$ albicans $=50$ casos; $C$.não albicans $=10$ casos 
Tabela 24-Distribuição da freqüência de candidíase sistêmica em 60 recémnascidos entre os anos de 1994 a 2003

\begin{tabular}{ccc}
\hline \hline Ano & Número de internações & TOTAL \\
N $(\%)$
\end{tabular}

Gráfico 4 - Distribuição das espécies de Candida encontradas em 60 recémnascidos com candidíase sistêmica -1994 a 2003

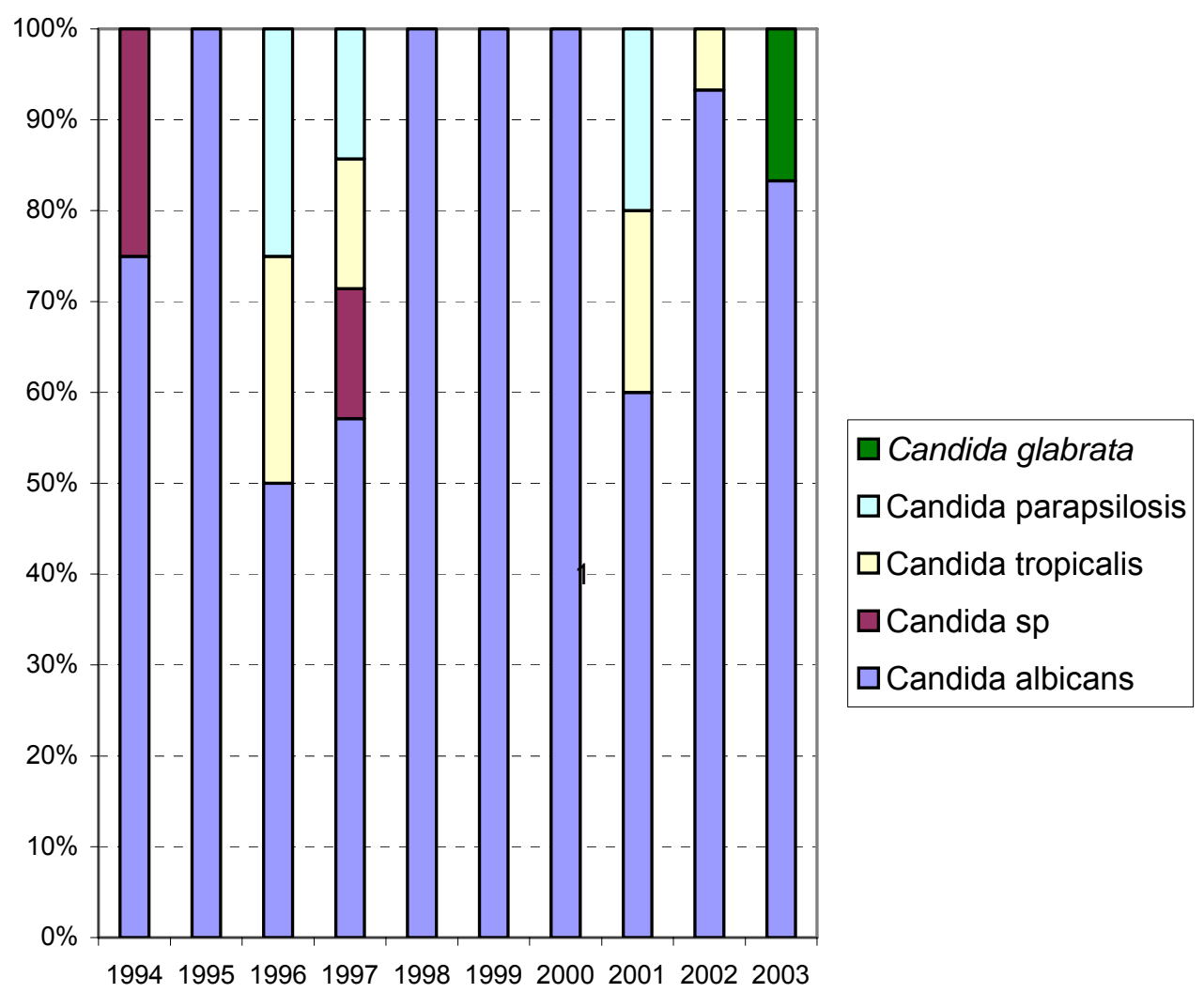

Ano da Infecção 
Na década de 1994 a 2003 houve 3219 admissões na UCINE, entre os quais 60 ( $1,8 \%$ dos casos) desenvolveram candidíase sistêmica, observando-se variação ampla, de 0,8\%, no ano de 1995 a 5,2\%, em 2002. Recentemente, outros autores relataram freqüências menores, utilizando os mesmos critérios diagnósticos de nosso estudo. Sastre et al. (2003), em estudo prospectivo multicêntrico incluindo 20565 neonatos, admitidos em 27 unidades neonatais na Espanha, relataram candidíase sistêmica em 0,57\% dos casos. Linder et al. (2003) em Israel, registraram a doença em 1,3\% de 4201 recém-nascidos admitidos em uma UTI neonatal. A freqüência de candidíase sistêmica é maior nos estudos que incluem somente recémnascidos de baixo peso, com variação de 2,2 a 12,9\% nos recém-nascidos com peso inferior a $1500 \mathrm{~g}$ e de $5,5 \%$ a $16,5 \%$ naqueles com menos de 1000 g (Johnson et al. 1984; Stoll et al 2002). Stoll et al. (2002), em estudo multicêntrico analisando a sepse de origem hospitalar em Unidade de Terapia Intensiva, durante o período de dois anos, incluindo 6956 neonatos com peso entre $401 \mathrm{~g}$ e $1500 \mathrm{~g}$, registraram candidíase sistêmica em 12\% dos casos. Os autores destacaram a importância atual da Candida albicans, que surgiu em terceiro lugar entre os microorganismos mais freqüentemente isolados. Guida et al. em (2003), em um estudo de coorte em 943 pacientes com peso inferior a 1500g, relataram sepse em 154 (16\%) pacientes, sendo 
identificada Candida sp. em $8 \%$ dos casos. Yinnon et al.(1992) relataram candidemia por Candida lusitaniae em um prematuro de $860 \mathrm{~g}$.

Em nossa casuística observamos predomínio de recém -nascidos prétermo, de baixo peso ao nascimento e do sexo masculino. A idade média ao diagnóstico da doença foi 31,6 dias. A associação de candidíase com prematuridade foi demonstrada em $65 \%$ dos casos, concordando com vários estudos (Isaacs et al. 1995; Stoll et al. 1996; Linder et al. 2003; Klingspor et al. 2004; Colby et al. 2004). Stoll et al. (1996), relataram infecção por Candida em 9\% dos episódios de sepse tardia em recém-nascidos de muito baixo peso. Em outro estudo incluindo 12 centros norte-americanos, os mesmos autores mostraram que a taxa de infecção hospitalar diminuiu significativamente com o aumento do peso de nascimento (Stoll et al. 2002). Linder et al. (2003), em um período de quatro anos, em Israel, relataram candidemia em 2,6\% em recém-nascidos de muito baixo peso e em 9,1\% daqueles de extremo baixo peso ao nascimento. Klingspor et al. (2004), na Suécia, entre 30 neonatos com candidíase sistêmica, observaram que 77\% apresentavam peso inferior a 1000 g. Colby et al. (2004) em casuística semelhante à nossa, incluindo 66 neonatos com candidemia, destacaram a ocorrência de peso de nascimento inferior a $1500 \mathrm{~g}$ em $71 \%$ dos casos, chamando a atenção a importância crescente desta patologia em recémnascidos de muito baixo peso.

A maior suscetibilidade dos recém-nascidos pré-termo à sepse fúngica pode ser explicada, em parte, pela imaturidade imunológica destes 
pacientes (Faix et al. 1989; Ng PC 1994; Karlowicz et al. 2002). Alguns autores analisaram a capacidade antifúngica mediada por linfócitos em recém-nascidos de baixo peso, prematuros e com doenças graves e demostraram que a capacidade de inibição linfocítica da Candida albicans em recém-nascidos pré-termo e de muito baixo peso é significativamente reduzida quando comparados com recém-nascidos de termo (Witek-Jausek et al. 2002).

Sabe-se que a taxa de infecção hospitalar em UTI neonatal é inversamente proporcional à idade gestacional. Stoll et al. (2002), analisando recém-nascidos pré-termo em UTI neonatal constataram que $46 \%$ dos neonatos com IG $<25$ semanas desenvolveram sepse tardia, fato observado em $29 \%$ quando a idade gestacional situou-se entre 25 a 28 semanas, $10 \%$ nos entre pacientes com idade gestacional entre 29 a 32 semanas e para somente $2 \%$ em recém- nascidos com idade gestacional superior a 32 semanas $(p<0,001)$. Em nossa casuística, nos pacientes com idade gestacional igual ou inferior a 28 semanas a taxa de candidíase sistêmica foi $25,6 \%$, entre 29 a 32 semanas foi $53,4 \%$ e em neonatos com idade gestacional superior a 32 semanas foi $20,5 \%$.

A maioria dos recém-nascidos apresentou peso inferior a $2500 \mathrm{~g}$, sendo $50 \%$ com peso inferior a $1500 \mathrm{~g}$ e $23,3 \%$ com peso inferior a $1000 \mathrm{~g}$. O predomínio de recém-nascidos de baixo peso de nascimento era esperado, devido à maior vulnerabilidade dessas crianças e concorda com os achados da literatura. Apesar do predomínio de recém-nascidos de baixo 
peso, houve uma porcentagem expressiva $(36,7 \%)$ de neonatos com peso superior a $2500 \mathrm{~g}$. Este comportamento pode ser explicado pela presença de malformações com indicação de procedimentos cirúrgicos na maioria destes pacientes, cuja principal indicação de internação na UTI neonatal foi a necessidade de cirurgia do aparelho digestivo, do sistema urinário ou sistema nervoso central. Estes dados são concordantes com estudo de Rabalais et al. (1996), que chamaram a atenção para o maior risco de candidíase sistêmica em neonatos com peso superior a 2500 g portadores de malformações congênitas, principalmente do trato gastrointestinal e cardíacas. Como a Candida habita normalmente o intestino humano, que constitui um reservatório do fungo, qualquer fator que provoque ruptura da barreira epitelial do intestino poderá ocasionar fungemia. O papel da colonização do trato gastrointestinal na patogênese da candidíase sistêmica foi muito bem destacado em um estudo de coorte multicêntrico realizado por Saiman et al. (2000), onde foram avaliados fatores de risco para candidemia em todos os recém-nascidos independentemente da idade gestacional, internados em UTI neonatal por período superior a 3 dias. Foram diagnosticados 35 casos de candidemia, sendo $43 \%$ destes precedidos pela colonização gastrointestinal, confirmada pelo estudo de tipagem molecular que possibilitou documentar a colonização prévia com o mesmo clone de fungo que causou a candidemia. Recentemente tem sido destacada a importância do jejum prolongado na patogênese da sepse fúngica em UTI neonatal. Recomenda-se que a dieta enteral mínima seja administrada para 
estes neonatos, com o objetivo de propiciar crescimento e maturação do intestino, utilizando-se preferencialmente, leite materno, devido a suas propriedades anti-infecciosas.

O predomínio do sexo masculino, observado por nós, concorda com outros estudos, que mostram ser a sepse bacteriana neonatal mais freqüente no sexo masculino. A maior suscetibilidade dos meninos é mais evidente nos casos de sepse causados por enterobactérias (Klein 2001). As causas para este comportamento ainda não são conhecidas. Carvalho et al. (2001) no Paraná, em 21 crianças com diagnóstico de infecção urinária por fungo, constataram que $81 \%$ dos pacientes eram do sexo masculino. No entanto, outros autores observaram freqüência semelhante entre os dois sexos na sepse fúngica (Paganini et al. 2000; Stoll et al. 2002; Benjamin et al. 2003a).

A idade média de 31 dias ao diagnóstico da infecção fúngica provavelmente pode ser atribuída a vários fatores. Sabe-se que a taxa de infecção hospitalar é diretamente relacionada ao aumento do tempo de internação. Os recém-nascidos pré-termo, com peso muito baixo ao nascer, freqüentemente necessitam de internação prolongada, para ganho de peso e monitorização de função cárdio-respiratória, ficando expostos ao risco de infecção. Os neonatos criticamente doentes, em tratamento para patologias já existentes, permanecem expostos por um período prolongado aos fatores de risco para sepse fúngica, notadamente aqueles mais perigosos, como uso de antibióticos, catéter venoso central e nutrição parenteral. Paganini et 
al. (2002) observaram que neonatos com catéter venoso central e uso de nutrição parenteral por período prolongado apresentaram maior probabilidade de desenvolver candidemia.

Observamos em nossa casuística que 96,6\% dos neonatos receberam tratamento com antibióticos antes do diagnóstico de candidíase sistêmica, por tempo médio variável de quatro a 17,6 dias, principalmente amicacina, vancomicina, penicilina cristalina, cefalosporinas de $3^{a}$ geração e carbapenens (Tabela 9). Além disso, constatamos a utilização freqüente de três ou mais antibióticos antes do diagnóstico de infecção fúngica. O uso prévio de antibióticos é citado por vários autores como um dos principais fatores de risco para infecção fúngica (Weese Mayer et al. 1987; Wey et al. 1989; Richet et al. 1991), Benjamin et al. (2000 e 2003 ) relataram forte associação entre o uso de cefalosporinas de $3^{a}$ geração ou o uso de carbapenem e candidemia. Costa et al. (2000) descreveram 86 casos de candidemia em adultos e crianças, sendo 10 recém-nascidos, dos quais $87 \%$ utilizaram vancomicina ou imipenem. Stoll et al. (2002) relataram o uso de vancomicina em $44 \%$ dos recém-nascidos de muito baixo peso com candidíase sistêmica, sendo o seu uso inversamente proporcional ao peso de nascimento. Este comportamento mostra a necessidade do uso criterioso de antibióticos em UTI neonatal.

O emprego de catéter venoso central é cada vez mais freqüente nas Unidades de Terapia Intensiva neonatais e esteve presente em 95\% de nossa casuística. Chien et al. (2002) em uma UTI no Canadá demonstraram 
que o estafilococo coagulase negativo e a Candida $s p$ foram os principais agentes envolvidos em infecções relacionadas ao catéter. A colonização da ponta do catéter e ou da pele no local da inserção, assim como a infusão de líquidos contaminados são mecanismos freqüentemente envolvidos na patogênese da infecção. A colonização da ponta do catéter é freqüente, podendo favorecer a ocorrência de embolia séptica, dificultando ainda mais o tratamento (Weese Mayer et al. 1987; Salzman et al. 1993; Cimolai et al. 2002). Farina et al. (1999) em estudo de 10 anos analisando candidemia em 168 pacientes adultos e crianças descreveram 26 neonatos, dos quais 25 tinham acesso venoso central. Em nossa casuística, entre os neonatos a cultura positiva do catéter, sangue e urina foi associada à positividade em $5 \%$ dos pacientes, urina e ponta de catéter em $1,7 \%$, no sangue e ponta de catéter em 3,3\%, no sangue, urina, ponta de catéter e líquido peritonial em 1,7\%. A emergência de Candida parapsilosis na sepse em UTI neonatal tem sido atribuída, em parte, à aderência do fungo à superfície do catéter venoso central. Aujard (2003), relatou candidemia em prematuros associada ao uso de catéter venoso central ou infecção cutânea, com predomínio de Candida parapsilosis em $60 \%$ dos casos. Silva et al. (2001) relataram três casos de candidemia por Candida parapsilosis em prematuros, todos com acesso venoso central.

A intubação traqueal foi o terceiro fator de risco mais freqüente em nosso estudo, ocorrendo em $91,7 \%$ dos casos. Sabe-se que este procedimento favorece a colonização endotraqueal e candidemia. 
A presença da cânula prejudica o mecanismo de limpeza mucociliar das vias aéreas e sua aspiração aumenta o risco de colonização do trato respiratório por patógenos (Rowen et al. 1994; Saiman et al. 2000).

A nutrição parenteral foi utilizada em $90 \%$ dos recém-nascidos, mostrando a importância deste fator de risco para candidíase sistêmica. A utilização de emulsão lipídica e soluções com altas concentrações de glicose favorecem o crescimento e multiplicação do fungo, sendo a nutrição parenteral considerada um fator de risco importante, independentemente da presença de catéter venoso (Weese Mayer et al. 1987; Saiman et al. 2000,). Guzmán et al. (2001) compararam recém-nascidos de muito baixo peso em uso de nutrição parenteral, considerando duas categorias de peso: inferior a $1000 \mathrm{~g}$ e entre $1000 \mathrm{~g}$ e $1250 \mathrm{~g}$. Os autores observaram que a incidência de complicações como displasia pulmonar, enterocolite necrosante e candidemia foi maior no grupo com peso inferior a $1000 \mathrm{~g}$. A candidemia ocorreu em 48\% dos RN com menos de $1000 \mathrm{~g}$ e 14,2\% no grupo com peso maior que $1250 \mathrm{~g}$.

Com relação à identificação de Candida spp em culturas, observamos que os locais de isolamento mais freqüentes foram sangue $(66,7 \%)$ e urina (66,7\%). Colby et al (2004) obtiveram resultados semelhantes, evidenciando cultura positiva para Candida sp no sangue em $40,9 \%$ dos casos, na urina em $30,3 \%$ e no sangue e urina em $15,2 \%$.

A espécie de Candida mais freqüentemente identificada foi Candida albicans, seguida de Candida tropicalis, Candida parapsilosis e Candida 
glabrata. Estes dados estão de acordo com a literatura, que destacam o predomínio de Candida albicans na candidíase sistêmica em adultos e crianças (Rennert et al. 2000; Macphail et al. 2002; Diekema et al. 2002; Tortorano et al. 2002; Kibbler et al.2003). Recentemente alguns estudos destacam a ocorrência freqüente de Candida parapsilosis em infecções neonatais. Paganini et al. (2002) descreveram Candida albicans em 50\% dos casos e Candida parapsilosis em 17\%. Stoll et al. (2002) identificaram Candida albicans em 5,8\% dos casos, Candida parapsilosis em 4,1\% e outras espécies em 2,3\%. Sastre et al. (2003) evidenciaram Candida albicans em 52,5\% dos pacientes, seguida de Candida parapsilosis em 23,7\% e Candida tropicalis em 7,6\%. Klingspor et al. (2004) descreveram Candida albicans em $67 \%$ dos casos, Candida glabrata em 15,7\% e Candida parapsilosis em 7\%. A freqüência de Candida parapsilosis obtida por estes autores foi semelhante à de nosso estudo onde esta espécie ocorreu em 5\% dos neonatos. Saxen et al. (1995), entre 58 neonatos infectados ou colonizados com Candida parapsilosis, destacaram a prematuridade como o maior fator de risco e associado a pior prognóstico, sendo o risco de óbito 16 vezes maior nos recém-nascidos pré-termo portadores de Candida parapsilosis em relação aos pacientes sem infecção . Lupetti et al. (2002) descreveram casos de transmissão horizontal de Candida parapsilosis em UTI neonatal. Faix (1992) destacaram a importância da colonização de catéter venoso por Candida parapsilosis. Kossoff et al. (1998) durante 15 anos, detectaram 111 casos de candidemia em neonatos, com predomínio 
de Candida albicans entre 1981 a 1990, e de Candida parapsilosis entre 1991 a 1995, destacando que a infecção por esta espécie ocorreu em 60\% dos casos e aumentou 11 vezes mais ao longo de 15 anos. Safdar et al. (2004) estudaram 75 pacientes, dentre estes 11 neonatos (15\%), destacando Candida albicans (37\%), Candida glabrata (31\%), Candida parapsilosis (17\%) e Candida tropicalis em 7\% dos pacientes. Hajjeh et al. (2004) evidenciaram Candida albicans em $60 \%$ dos casos de candidemia, seguidos por Candida parapsilosis em $27 \%$ e Candida glabrata em $13 \%$. Duran et al. (2003) em estudo retrospectivo da suscetibilidade antifúngica em culturas por um período de 5 anos (1997-2001), identificaram Candida parapsilosis em $41,5 \%$ dos casos, Candida albicans em 35,8\%, Candida glabrata em 9,4\%,Candida krusei em 5,5\%, Candida tropicalis em 3,7\% e Candida guilliermondii em 3,7\%. A Candida parapsilosis foi a espécie mais comumente observada no berçário. Huang et al. (2000) compararam 22 casos de candidemia por Candida parapsilosis e 24 casos de candidemia por Candida albicans durante um período de 3 anos, observando maior gravidade e disseminação da doença nas infecções por Candida albicans , cujos pacientes mostraram maior frequência de hipoxemia e insuficiência respiratória, requerendo períodos prolongados de intubação, episódios de bradicardia e maior tempo de permanência de catéter vascular. Fairchild et al. (2002) relataram 58 casos de candidemia em unidade de terapia intensiva neonatal durante um período de 8 anos, sendo 15\% causados por Candida glabrata. Os autores observaram que, em relação às outras espécies, a 
Candida glabrata ocorreu em recém-nascidos com maior idade gestacional e maior peso de nascimento.

Os sinais e sintomas mais freqüentemente observados no momento da suspeita de candidíase sistêmica foram: alterações respiratórias (estertores pulmonares, piora radiológica pulmonar), anormalidades da temperatura (hipotermia e hipertermia), letargia, hepatomegalia e esplenomegalia. Estes achados já foram descritos por vários autores. Em estudo retrospectivo de 49 pacientes com sepse por Candida em UTI neonatal, Makhoul et al. (2001) descreveram a hipertermia como uma manifestação clínica freqüente, ocorrendo em $42,8 \%$ dos pacientes e em nosso estudo foi responsável por $63,3 \%$ dos casos.

Observamos monilíase oral em $20 \%$ dos neonatos, concordando com vários relatos de associação entre candidíase mucocutânea e candidíase invasiva. Faix et al. (1989), em um estudo prospectivo em 358 pacientes com peso de nascimento inferior a $1500 \mathrm{~g}$ encontrou candidíase mucocutânea em 28 (7,8\%), candidíase invasiva em 16 (4,5\%), dos 28 pacientes com candidíase mucocutânea, 9 (32\%) desenvolveram candidíase invasiva apesar do tratamento específico, mostrou que pacientes de muito baixo peso que tinham candidíase mucocutânea têm um risco significativamente maior de desenvolver candidíase invasiva que pacientes sem candidíase mucocutânea. Winter et al. (1994) relataram um caso de um recém-nascido prematuro com sinais de candidíase congênita, mãe com coriamnionite por Candida. Arnavielle et al. (2000) descreveram 3 casos de 
candidemia por Candida albicans em mães assintomáticas. Em estudo de Gargneur et al. (2001) em recém-nascidos porém, ocorreu baixa colonização e infecção causada por Candida parapsilosis.

As complicações em diferentes órgãos ou sistemas foram observadas na maioria dos casos (85\%), conforme era esperado, considerando a gravidade da infecção fúngica em recém-nascidos. Nestes pacientes a candidemia costuma se associada a complicações graves, que tornam o prognóstico particularmente sombrio.

A principal complicação multissistêmica observada por nós foi infecção do trato urinário concordando com os estudos da literatura que destacam a importância do comprometimento do sistema urinário na sepse por Candida spp. A candidíase do sistema urinário pode se manifestar como micetoma fúngico e uropatia obstrutiva (Al-Rasheed 1994). O abscesso renal foi observado somente em um $(1,7 \%)$ neonato do nosso estudo, que apresentava também malformação do uretér. Makhoul et al. (2001) relataram abscesso renal em $7,1 \%$ dos casos. A presença de malformação do sistema urinário, observada em $5 \%$ dos nossos pacientes, constitui um fator adicional para a infecção fúngica. Bryant et al. (1999) descreveram candidíase renal em pacientes com candidúria, com presença de micetoma ou abscesso renal em $42 \%$ dos pacientes. Karpman et al. (2003) relataram um neonato de termo com massa renal e diagnóstico de hidronefrose. Benjamin et al. (1999) em 30 neonatos com candidemia, que realizaram ultra-sonografia do sistema urinário, relataram a presença de micetoma em 
33\% dos casos. Entre 14 neonatos com micetoma renal sem obstrução completa, 3 apresentavam anormalidades do trato urinário. Bibiloni et al. (2001) descreveram 7 casos de micetoma fúngico em neonatos com malformações do trato urinário. A presença de bexiga neurogênica, em neonatos com mielomeningocele, observada em $7 \%$ da nossa casuística, também resulta no aumento do risco de infecção fúngica. Carvalho et al. (2001) entre 21 crianças com candidúria, observaram malformação do trato urinário e mielomeningocele em $24 \%$ dos casos, respectivamente. Yoo et al. (1995) descreveram casos de falência renal causado por micetoma fúngico. Hitchcock et al. (1995) relataram 5 neonatos com trato urinário, nos quais a obstrução do trato urinário devido à malformação congênita foi identificada como em 3 casos e, em dois casos houve formação de micetoma fúngico. Noyola et al. (2001), relataram anormalidades ao ultra-som do sistema urinário em 7,7\% dos neonatos.

A pneumonia foi a segunda complicação mais freqüente, observada em $56,7 \%$ dos recém-nascidos. A associação significativa entre pneumonia e óbito sugere que esta complicação contribuiu para agravar o prognóstico da candidíase. O diagnóstico de pneumonia por Candida apresenta dificuldades, já que o isolamento do fungo em secreção traqueal não é indicativo de pneumonia fúngica, podendo significar somente colonização do sistema respiratório. A maioria dos neonatos em nosso estudo necessitou de intubação traqueal prolongada para suporte ventilatório, sendo provável que a presença deste fator de risco tenha contribuído para a presença de 
pneumonia e mortalidade maior entre estes pacientes. Nossos achados estão de acordo com outros autores, que descreveram freqüência elevada de pneumonia em UTI neonatal, utilizando critérios diagnósticos semelhantes aos do nosso estudo. Reyes et al. (1996) relataram imagem radiográfica de broncopneumonia em $57 \%$ dos pacientes estudados. Chowdhary et al. (2003) em 16 neonatos com candidemia por Candida tropicalis, diagnosticaram insuficiência respiratória em 62,5\% dos casos. Sob o ponto de vista fisiopatológico, a pneumonia por Candida, pode ocorrer também após aspiração de secreção vaginal infectada pelo feto, durante o nascimento ou por transmissão hematogênica ou embólica (Baley, 1991). Mazor et al. (1993), descreveram uma gestante com infecção assintomática do líquido amniótico por Candida sp, com nascimento de recém-nascido prétermo, com necessidade de ventilação mecânica e imagem radiológica de pneumonia.

A meningite ocorreu em nossos pacientes em 13,3\% dos casos, sendo a identificação de Candida no líquor pouco freqüente. Entre oito neonatos com exame quimiocitológico do líquor anormal, apenas três mostraram crescimento de fungo no líquor, confirmando a dificuldade do diagnóstico de meningite por Candida através da cultura de líquor (Chen et al. 2004). Moreno et al. (1994) em estudo de 577 neonatos com sepse e ou meningite, durante um período de 18 anos entre 107 casos de meningite, identificaram Candida sp no LCR em somente 1,9\% dos casos. Por esta razão, concordamos com os autores que recomendam considerar meningite 
fúngica qualquer anormalidade do exame de LCR em neonatos com hemocultura positiva para fungo (Baley 1991). Diferentemente do nosso estudo, Fernandez et al. (2000) em estudo retrospectivo de 10 anos, de 106 neonatos evidenciaram meningite em 23 pacientes $(21,7 \%)$, com cultura de líquor positiva na maioria dos casos (74\%). A pleocitose ocorreu em $39 \%$ dos recém-nascidos e a hipoglicorraquia em 25\%. Os autores destacaram que valores quimiocitológicos normais não excluem o diagnóstico de meningite fúngica, podendo ocorrer crescimento do fungo no LCR nestas condições. O comprometimento do sistema nervoso central provavelmente é mais freqüente entre recém-nascidos pré-termo de muito baixo peso. Arisoy et al. (1994), em estudo de meningite fúngica em crianças maiores e neonatos, relataram nove pacientes recém-nascidos com presença de Candida albicans em pelo menos uma cultura do líquor, sendo 8 prematuros de muito baixo peso. A presença de malformação do sistema nervoso ou derivação ventrículo-peritonial é outro fator que aumenta o risco de meningite fúngica. Chiou et al. (1994) em estudo retrospectivo, observaram infecção por Candida spp em válvula de derivação ventriculoperitonial em 17\% de 48 lactentes. Em nossa casuística entre os neonatos com meningite, 12,5\% apresentavam hidrocefalia com derivação ventrículo-peritonial.

Para melhorar o diagnóstico de meningite por Candida sp a pesquisa do antígeno mannan no LCR foi utilizada por alguns autores. Lunel et al. (2004) em cinco pacientes com candidíase sistêmica, detectaram a presença do antígeno mannan em quatro. Ng et al. (2000) destacaram o aumento 
liquórico das concentrações de citocinas em neonatos sem anormalidades bioquímicas com sepse por Candida sp.

A endocardite, observada em $13,3 \%$ dos nossos pacientes, era antigamente considerada uma doença rara. Com o advento da ecocardiografia tornou-se possível detectar vegetações e/ou trombos no exame cárdio-vascular. Trata-se de uma complicação cuja mortalidade é elevada, com piora considerável do prognóstico da infecção fúngica quando há comprometimento cardíaco. Sua patogênese é complexa e, na maioria dos casos, envolve trauma do endocárdio ou do endotélio vascular, expondo o colágeno subendotelial, com aderência de plaquetas ao colágeno e depósito de fibrina com formação de trombo. Krebs et al. (1999b) relataram um caso de um recém-nascido com 11 dias de vida que apresentou endocardite associada a múltiplas malformações congênitas e cateterismo venoso central. Nos nossos pacientes o principal fator de risco para a ocorrência de endocardite foi a presença de catéter venoso central.Nenhum neonato apresentava cardiopatia congênita complexa. Rios et al. (1993) em estudo semelhante ao nosso relataram $15(13,6 \%)$ casos de endocardite por Candida spp no México entre 1980 e 1991. Todos os pacientes apresentavam catéter venoso central e $20 \%$ eram portadores de cardiopatia congênita. Vricella et al. (2003) descreveram um caso de obstrução ventricular direita causada pela presença de uma massa vegetante causando colapso cardiocirculatório. Linder et al. (2000) descreveram um prematuro de 24 semanas com massa cardíaca em átrio direito. Daftary et 
al. (1999) relataram quatro casos de massa fúngica intracardíaca em prematuros extremos tratados cirurgicamente, que faleceram, apesar do tratamento com anfotericina liposomal na dose de $6 \mathrm{mg} / \mathrm{Kg} / \mathrm{dia}$. Vaideeswar et al. (1994) relataram um neonato com massa fúngica intracardíaca no lado esquerdo à necrópsia. Divekar et al. (2004) relataram endocardite em prematuro com estenose aórtica e sepse por Candida parapsilosis . A trombose venosa profunda ocorreu em 10\% dos neonatos da nossa casuística, sendo esta porcentagem bastante elevada, considerando-se a que a trombose de grandes vasos é pouco freqüente no período neonatal. Este achado indica que a presença do catéter associado à infecção fúngica resultaram em lesão endotelial, depósito de fibrina e formação de trombo. Outros autores relataram freqüência de trombose ainda mais alta do que a observada por nós. Noyola et al. (2001), entre 65 neonatos, observaram a presença de trombo ou vegetação em 15,2\%.

A endoftalmite, observada em 6,7\% dos recém-nascidos, é uma complicação da candidíase sistêmica neonatal citada em frequência variável de zero (Donahue et al. 2003) a 50\% dos casos (Baley et al. 1981, Deutsch et al. 1989). A lesão pode se manifestar como uma formação arredondada, branca, de aspecto algodonoso, que flutua no humor vítreo (Baley et al. 1984; Butler et al. 1990; Faix 1992; Lee et al. 1998). A frequência observada por nós foi semelhante à de Noyola et al. (2001), que descreveram endoftalmite em $6 \%$ entre 67 neonatos com candidíase no período de 10 anos. Johnston et al. (2000), descreveram um recém-nascido com 
candidíase sistêmica e formação de catarata. Stern et al. (2001) relataram um caso de endoftalmite por Candida quatro meses após o término do tratamento antifúngico. Droham et al. (2002) relataram um prematuro de 28 semanas com endoftalmite e abscesso, submetido a tratamento cirúrgico. Shah et al. (2000), relataram um caso de abscesso intralenticular em prematuro de 29 semanas com candidemia.

Alguns autores têm descrito associação entre coriorretinite fúngica e retinopatia da prematuridade. Noyola et al. (2002) compararam 46 neonatos com candidemia e 39 sem candidemia, todos com peso inferior a $1500 \mathrm{~g} \mathrm{e}$ idade gestacional menor que 28 semanas. Nos neonatos com candidemia a retinopatia da prematuridade, foi observada em $93,5 \%$ dos casos e em $84,8 \%$ dos neonatos sem candidemia. A necessidade de procedimento cirúrgico para retinopatia foi significativamente maior $(p=0,008)$, entre os recém-nascidos com candidemia indicando que o comprometimento ocular foi de maior gravidade nestes neonatos. Gago et al. (2002) descreveram um caso de retinopatia da prematuridade estágio 3 e endoftalmite por Candida em um prematuro com idade gestacional de 23 semanas. Por outro lado, Karlowicz et al. (2000b) em 449 prematuros com peso inferior a $1000 \mathrm{~g}$ concluíram que a candidemia não foi um fator de risco independente para o desenvolvimento de retinopatia da prematuridade.

Não houve associação estatisticamente significante entre espécie de fungo e evolução clínica, fato que pode ser atribuído em parte, ao pequeno número de neonatos com infecção por Candida não albicans. 
Com relação ao tipo de tratamento, observamos que a maioria dos recém-nascidos recebeu tratamento com formulação lipídica de Anfotericina B, sendo a sobrevida significativamente maior neste grupo de crianças, sugerindo maior eficácia terapêutica das formulações lipídicas em relação à Anfotericina B convencional.

A anfotericina B convencional, amplamente utilizada desde 1956, é um macrolídeo polieno, que adere ao ergosterol da membrana celular do fungo, levando à alteração da sua permeabilidade, com lise e morte celular. Devido à má absorção gastrointestinal, existe somente sob a forma parenteral, apresentando boa distribuição nos tecidos, especialmente no fígado, e baço. Trata-se de medicamento nefrotóxico, que pode causar alterações eletrolíticas e efeitos cardiotóxicos. A nefrotoxicidade é cumulativa e caracteriza-se por resistência ao hormônio antidiurético, com poliúria, acidose tubular renal, hipocalemia e falência renal. Em neonatos, as alterações da função renal, traduzidas por aumento da uréia plasmática, diminuição do clearance de creatinina e anormalidades de eletrólitos, geralmente são reversíveis, sendo às vezes difícil estabelecer se estas alterações são decorrentes do tratamento ou da própria doença (Baley et al. 1984, Frattarelli et al. 2004, Dean et al. 1994). Holler (2004) estudaram 573 recém-nascidos com peso inferior a 1250 g com candídíase sistêmica tratados com anfotericina B entre os anos de 1998 a 2000, constatando que 4,4\% apresentaram sepse fúngica e, dentre estes $44 \%$ evoluíram com comprometimento da função renal. Glick et al. (1993) em estudo 
retrospectivo de 36 neonatos com peso superior a 1000 gramas, com cultura positiva para Candida spp, tratados com anfotericina B convencional com dose inicial de $0,5 \mathrm{mg} / \mathrm{kg} / \mathrm{dia}$ seguida do aumento para $1 \mathrm{mg} / \mathrm{kg} / \mathrm{dia}$ não observaram alterações renais.

Vários estudos relataram casos de sepse por Candida spp. com complicações, como meningite causada por Candida tropicalis (Ahuja et al. 2003), ventriculite (Murphy et al. 2000), abscesso cerebral por Candida albicans(Kamitsuka et al. 1995), uropatia obstrutiva (Mcdonnell et al. 1995), micetoma e meningite (Abraham et al. 2002), sem registro de toxicidade do tratamento. Anaisse et al. (1996) em estudo randomizado multicêntrico, comparando pacientes que utilizaram anfotericina B convencional e fluconazol, não observaram diferenças em relação à sobrevida, porém registraram taxa maior de efeitos adversos com o uso de anfotericina B (35\%) do que com o uso de fluconazol (5\%).

As novas formulações lipídicas da anfotericina $B$ foram desenvolvidas com o intuito de possibilitar a administração de doses elevadas de anfotericina, atingindo rapidamente concentrações séricas terapêuticas, com menor toxicidade. As vesículas liposomais facilitam a penetração do antifúngico no sistema reticuloendotelial e melhoram o clearance na circulação sistêmica. Os primeiros estudos mostrando resultados do uso de preparado lipídico de anfotericina em neonatos surgiram no início da década de 1990. Lackner et al. (1992) relataram o uso de anfotericina liposomal em dois neonatos de muito baixo peso, que 
receberam doses elevadas desta medicação sem alterações eletrolíticas, renais ou hepáticas. Jarlov et al. (1995) descreveram o primeiro caso de meningite por Candida albicans em um prematuro com 27 semanas de idade gestacional, tratado com anfotericina liposomal com êxito. Weitkamp et al. (1998), relataram 21 prematuros que receberam anfotericina liposomal em doses elevadas com boa eficácia clínica e sem efeitos colaterais. Krebs et al.(1999a), em 11 neonatos admitidos na UTI neonatal, tratados com formulação lipídica da anfotericina (dispersão coloidal ou anfotericina liposomal) observaram menos efeitos colaterais e menor duração do tratamento quando comparados com 10 neonatos que receberam anfotericina convencional. Juster-Reicher et al. (2000) relataram 24 neonatos de muito baixo peso tratados com anfotericina liposomal, com erradicação da doença em 92\% dos episódios. Stocker et al. (2000) relataram um prematuro de 26 semanas com micetoma bilateral tratado com anfotericina B liposomal e fluconazol e Jorge et al. 2003 também trataram com anfotericina liposomal um prematuro extremo com meningite por Candida com sucesso. Linder et al. (2003), estudaram neonatos com candidíase sistêmica selecionados para tratamento com anfotericina B convencional ou anfotericina liposomal ou dispersão coloidal, conforme a concentração de creatinina plasmática. Os preparados lipídicos foram utilizados sempre que a creatinina foi superior a $1,2 \mathrm{mg} / \mathrm{dl}$, não tendo sido observada diferença de mortalidade entre os grupos. Houve esterilização no sangue em $67,6 \%$ dos pacientes que receberam anfotericina $B$, em $83,3 \%$ dos casos que utilizaram 
anfotericina liposomal e em $57,1 \%$ dos que receberam dispersão coloidal de anfotericina. Leibovitz (2002) em revisão da literatura sobre o tema, relatou três estudos não controlados em neonatos com candidíase sistêmica utilizando doses de anfotericina lipídica de 1 a $7 \mathrm{mg} / \mathrm{kg} / \mathrm{d}$, concluindo que o medicamento é efetivo e seguro. Favel et al. (2003) relataram um caso de infecção renal por Candida lusitaniae tratado com anfotericina liposomal e fluconazol sem sucesso.Juster-Reicher et al. (2003) em 37 recém-nascidos com candidíase sistêmica tratados com doses elevadas de anfotericina liposomal de 5 a $7 \mathrm{mg} / \mathrm{kg} / \mathrm{dia}$, relataram maior rapidez na erradicação da doença. Sastre et al. (2003) em estudo multicêntrico na Espanha estudaram 118 recém-nascidos com candidíase sistêmica, dos quais a maioria (68,6\%) foi tratada com anfotericina lipídica e somente sete receberam anfotericina B convencional, sendo o tratamento suspenso em três destes devido à nefrotoxicidade. Os autores não observaram diferença de mortalidade e incidência de efeitos colaterais entre as diferentes formulações lipídicas.

Apesar do resultado observado por nós e dos estudos citados sobre a eficácia dos preparados lipídicos, até o momento, não existem estudos controlados demonstrando que sua utilização resulta em diminuição da mortalidade por sepse fúngica neonatal. Em nossa opinião, considerando a relativa limitação orgânica do recém-nascido pré-termo em se adaptar a situações de estresse, deve-se escolher sempre medicamentos que acarretem o menor risco possível de iatrogenia. Seria, portanto, recomendável prescrever preparado lipídico de anfotericina nestes recém- 
nascidos para prevenir o aparecimento de efeitos indesejáveis do tratamento, principalmente insuficiência renal e hepática. A maior limitação para o seu uso é o custo elevado do medicamento. Kotwani et al. (2003), descreveram o uso de uma nova formulação lipídica de anfotericina B (LAMP-LCR-1), de menor custo, utilizada em 23 neonatos com candidíase, 14 dos quais responderam completamente ao tratamento com 1mg/kg/dia por 28 dias.

Observamos que a mortalidade global dos neonatos internados na UTI durante a década estudada foi 10,9\%, enquanto nos recém-nascidos com candidíase foi 33,3\%, estando significativamente associada à presença de pneumonia e à plaquetopenia. Vários autores mostram taxas elevadas de mortalidade na candidíase sistêmica, concordando com os resultados do presente estudo, com taxas variáveis de 10,2\% (Sastre et al. 2003) a 31\% (Stoll et al. 2002, Chapman 2003; Klingspor et al. 2004). A mortalidade elevada reflete a gravidade da doença, principalmente quando ocorrem complicações multissistêmicas graves, como a presença de plaquetopenia persistente ou pneumonia observadas nos pacientes que faleceram. A associação entre pneumonia e óbito, conforme já foi discutido, confirma a gravidade desta complicação em neonatos com candidíase. A importância da plaquetopenia na sepse fúngica neonatal foi destacada por Guida et al. (2003), que demonstraram contagem plaquetária inicial significamente menor, nadir mais baixo, maior incidência e maior duração da trombocitopenia na sepse fúngica e na sepse por bactérias gram-negativas, 
quando comparada à sepse por bactérias gram-positivas. Da mesma forma, Benjamin et al. (2000), mostraram que a sepse fúngica está associada com freqüência maior plaquetopenia do que a sepse por Staphylococcus coagulase negativo em recém-nascidos. Warris et al. (2001) compararam oito recém-nascidos pré-termo com candidemia com um grupo controle constituído por 16 neonatos portadores de sepse bacteriana, constatando plaquetopenia em $100 \%$ dos pacientes com infecção por Candida sp e em apenas 50\% dos neonatos do grupo controle.

Concluímos que a freqüência de complicações da candidíase sistêmica na presente casuística foi elevada, com lesões detectadas em diversos órgãos e sistemas, que provavelmente contribuíram para a alta mortalidade da população estudada. Por se tratar de infecção que acomete preferencialmente recém-nascido pré-termo, portador de fatores de risco inerentes ao seu tratamento clínico, é de grande importância considerar cuidadosamente a indicação de procedimentos invasivos e do uso de medicamentos, especialmente antibióticos, nessas crianças. 
Conclusões 
O presente estudo permitiu-nos chegar às seguintes conclusões:

- A freqüência de candidíase sistêmica na Unidade de Cuidados Intensivos Neonatal do ICr-HCFMUSP no período de 1994 a 2003 foi alta $(1,8 \%)$, variando de $0,8 \%$, a $5,1 \%$, sendo estas taxas superiores às observadas recentemente por outros autores.

- Os sinais e sintomas mais freqüentes foram os mesmos observados na sepse bacteriana, com destaque para as alterações respiratórias, anormalidades da temperatura, letargia e hepatoesplenomegalia.

- A espécie de Candida mais freqüentemente identificada foi Candida albicans, em concordância com a maioria dos estudos publicados sobre o tema. Em uma menor proporção de casos foram identificadas Candida tropicalis, Candida parapsilosis ou Candida glabrata. 
- As complicações multissistêmicas foram freqüentes e graves, com mortalidade elevada (33,3\%), associada significativamente à ocorrência de pneumonia ou plaquetopenia.

- A sobrevida foi significativamente maior no grupo que recebeu formulação lipídica da Anfotericina B, sugerindo maior eficácia deste tipo de tratamento. 


\section{Referências}

Esta dissertação está de acordo com:

Referências: adaptado de International Committee of Medical Journals Editors (Vancouver)

Universidade de São Paulo.Faculdade de Medicina. Serviço de Biblioteca e Documentação.

Guia de apresentação de dissertações, teses e monografias. Elaborado por Anneliese Carneiro da Cunha,

Maria Júlia de A. L. Freddi, Maria F. Crestana, Marinalva de Souza Aragão, Suely Campos Cardoso, Valéria

Vilhena. São Paulo: Serviço de Biblioteca e Documentação;2004.

Abreviaturas dos títulos dos periódicos de acordo com List of Journals Indexed in Index Medicus. 
Abraham M, Mampilly T, Paul JP, FVJ. Renal Failure from obstrutive Fungal Mycetoma and Fungal Sepsis in na Infant. Indian Pediatr. 2002;39:769-72.

Ahuja SR, Karande S, Kulkarni MV, Tendolkar.Candida tropicalis meningitis in a Young infant. Ind J Pediatr. 2003;70:925-7.

Al-Rasheed SA. The management of fungal obstructive uropathy in neonates and infants. Ann Trop Paediatr. 1994;14:169-75.

Anaissie EJ, Darouiche O, Abi-Said D, Uzun O, Mera J, Gentry LO, Williams T, Kontoyiannis DP, Karl CL, Bodey GP. Management of Invasive Candidal Infections: Results of a Prospective, Randomized, Multicenter Study of Fluconazole Versus Amphotericin B and Review of the Literature. Clin Infect Dis. 1996;964-72.

Anker LNVD, Popele NMLV, Sauer PJJ. Antifungal Agents in Neonatal Systemic Candidiasis.Antimicrob. Agents Chemother.1995:1391-7.

Arisoy ES, Arisoy AE,Jr WMD. Clinical significance of fungi isolated from cerebrospinal fluid in children. Pediatr Infect Dis J. 1994;13:128-33. 
Arnavielhe S, Blancard A, Mallie M, Bastide JM .Case Report of Three Candida albicans Infections Detected at Delivery. J Infect. 2000;40:88-96.

Aujard Y, Farnoux C, Lefevre S, Maury L, Delezoide AL, Maiani-Kurkdjian P.Infections néonatales à Candida. Arch Pédiatr 2003;10:569-74.

Baley JE, Annabe WL, Kliegman RM. Candida endophtalmitis in the premature infant. J Pediatr. 1981;98:458-61.

Baley JE, Kliegman, RM, Fanaroff AA. Disseminated fungal infection in very low birth-weight infants.; Pediatrics. 1984;73:144-52.

Baley JE. Neonatal Candidiasis: The current challenge. Clin Perinatol. 1991;18:263-80.

Benjamin DK Jr, Ross K,Mc Kinney RE Jr, Benjamin DK, Auten R , Fisher RG. When to suspect fungal infection in neonates :clinical comparison of Candida parapsilosis fungemia with Coagulase negative staphylococcal bacteremia. Pediatrics. 2000;106:712-18.

Benjamin DK Jr,Fisher RG, McKinney RE Jr, et al:Candidal mycetoma in the neonatal kidney. Pediatrics.1999;104:1126-29 
Benjamin DK Jr, Garges H, Steinbach WJ. Candida bloodstream infection in neonates. Semin Perinatol. 2003a;27:375-83.

Benjamin D K Jr, Poole C, Steinbach Rowen JL, Walsh TJ. Neonatal Candidemia and End -Organ Damage: A critical Appraisal of the Literature Using Meta-analytic Techniques. Pediatrics. 2003b;112:634-40.

Benjamin DK Jr, DeLong ER, Steinback WJ, Cotton CM, Walsh TJ, Clark RH. Empirical Therapy for Neonatal Candidemia in Very Low Birth Weight Infants. Pediatrics. 2003c;112:543-47.

Bennet JE. Antifungal agents. In: Hardman and Gilman̉s the pharmalogical basis of therapeutics.10th ed. New York: Mc Graw-Hill; 2001. p.1295-312.

Bibiloni N, Spizzirri F, Zalba J. Successful conservative treatment of severe renal candidiasis with fungus balls. Pediatr Nephrol. 2001;16:394.

Brandt ME,Benjamin LE,Steinkraus GE. Pseudooutbreak of Candida Versatilitis fungemia in a microbiology laboratory. Diagn Microbiol Infect Dis. 2003;46:73-5

Bryant K, Maxfield C, Rabalais G. Renal candidiasis in neonates with candidúria. Pediatr Infect Dis J. 1999;18:959-63. 
Butler KM, Baker CJ:Candida: An increasingly important pathogen in the nursery. Pediatr Clin North Am. 1988;35;543.

Campbell JR,Zaccaria E, Baker CJ.Systemic Candidiasis in Extremely Low Birth Weight Infants Receiving Topical Petrolatum Ointment for Skin Care:A Case -Control Study. Pediatrics. 2000;105:1041-5.

Carvalho M, Guimarães C M, Mayer JR Jr, Bordignon GPF, Telles\Q.Hospital -Associated Funguria: Analysis of risk factors, Clinical Presentation and Outcome. Braz J Infect Dis. 2001;5:313-18.

Chapman RL, Faix RG. Persistently positive cultures and outcome in invasive neonatal candidiasis. Pediatr Infect Dis J. 2000;19:822-7.

Chapman RL. Candida infections in the neonate. Curr Opin Pediatr. 2003;15:97-102.

Chen T, Chen HP, Fung CP, Lin MY, Yu W, Liu CY. Clinical characteristics, treatment and prognostic factors of candidal meningitis in a teaching hospital in Taiwan. Scand J Infect Dis. 2004;36:124-30 . 
Chiang AKS, Chan GCF, Ma SK, Ng YK ,Ha SY,Lau YL. Disseminated Fungal Infection Associated with Myeloperoxidase Deficiency in a Premature neonate. Pediatr Infect Dis. 2000;19:1027-9.

Chien L, Macnab Y, Aziz K, Andrews W, McMillan DD, Lee SK et al.The Canadian Neonatal Network. Variations in central venous catheter-related infection risks among Canadian neonatal intensive care units. Pediatr Infect Dis J. 2002;21:505-11.

Chiou CC,Wong TT, Lin $\mathrm{HH}$, Hwang $\mathrm{B}$, Tang RB,Wu KG, Lee BH.Fungal Infection of Ventriculoperitoneal Shunts in Children. Clin Infect Dis. 1994;19:1049-53.

Chowdhary A,Becker K, Fegeler W, Gugnani HC,Kapoor L, Randhawa VS,Mehta G. An outbreak of candidemia due to Candida torpicalis in a neonatal intensive care unit. Mycoses. 2003;46:269-74.

Cimolai N, Davis J, Trombley C. Candida dubliniensis Fungemia and Vascular Acess Infection. J Pediat Hematol Oncol. 2002;24:237-9.

Colby CE, Drohan L, Benitz W, Hintz SR. Low Yield of Ancillary Diagnostic Studies in Studies in Neonates Infected with Candida. J Perinatol. 2004; 24:241-6. 
Colombo AL, Nucci M, Salomão R, Branchini MLM, Richtmann R, Derossi A, Wey SB. High Rate of Non-albicans candidemia in Brazilian Terciary Care Hospitals. Diagn Microbiol Infect Dis. 1999; 34:281-6.

Costa SF, Marinho I, Araújo EAP, Manrique AEI, Medeiros EAS, Levin AS.Nosocomial fungaemia: a 2 year prospective study. J Hosp Infect. 2000; 45: 69-2.

Daftary AS, Palote SK, Whitehall JS.Intracardiac fungal masses in high-risk neonates: clinical observations. Acta Paediatr. 1999; 88: 1009-13.

Darmstadt GL, Dinulos JG, Miller Z. Congenital cutaneous candidiasis: clinical presentation, patogénesis, and management Guidelines. Pediatrics. 2000; 105:438-44.

Dean JL, Wolf JE, Ranzini AC, Laughlin MA. Use of amphotericin B during pregnancy : case report and review. Clin Infect Dis. 1994;18:364-8.

Deutsch D. Adler S, Teler J, Savir H. Endogenous candidal endophthalmitis. Ann Ophthalmol.1989;73:144-52.

Diekema DJ, Messer SA, Brueggemann AB, Coffman SL, Doern GV, Herwaldt LA, Pfaller MA. Epidemiology of candidemia: 3-Year results from 
emerging infections and the epidemiology of lowa organisms study. J Clin Microbiol. 2002;1298-302.

Divekar A, Rebekya IM, Soni R.Late Onset Candida parapsilosis Endocarditis After Surviving Nosocomial Candidaemia in an Infant with Structural Heart Disease. Pediatr Infect Dis J. 2004; 23:472-4.

Doerr CA,Demmler GJ, Garcia -Prats, Brandt ML. Solitary pyogenic liver abscess in neonates: report of three cases and review of the literature. Pediatr Infect Dis J. 1994; 13:64-9.

Donahue SP, Hein E, Sinatra RB. Ocular involvement in children with candidaemia. Am J Ophthalmol. 2003;135:886-7.

Drohan L, Colby CE, Brindle ME, Sanislo S, Ariagno RL. Candida (Amphotericin -Sensitive) Lens Abscess Associated With Decreasing Arterial Blood Flow in a Very Low Birth Weight Preterm Infant. Pediatrics. 2002;110:1-4.

Duran MT, VelascoD,Canle D,Moure R, Villanueva R. Susceptibilidad antifúngica de aislados de Candida spp. de hemocultivos en un período de cinco años(1997-2001). Enferm Infecc Microbiol Clin. 2003;21: 488-92. 
Dyke MP, Ott K: Severe thrombocytopenia in extremely low birthweight infants with systemic candidiasis. J Paediatr Child Health. 1993;29:298-301.

Fairchild KD, Tomkoria S , Sharp EC,Mena FV. Neonatal Candida glabrata sepsis:clinical and laboratory features compared with other Candida species. Pediatr Infect Dis J. 2002;21:39-43.

Faix RG, Kovarik SM, Shaw TR, Johnson RV. Mucocutaneous and invasive candidiasis among very low birth weight $(<1500 \mathrm{~g})$ infants in intensive care nurseries: A prospective study. Pediatrics.1989; 83:101-7.

Faix RG. Systemic Candida infections in infants in intensive care nurseries: High incidence of central nervous system involvement. J Pediatr. 1984; 105:616.

Faix RG.Invasive neonatal candidiasis: comparison of albicans and parapsilosis infection. Pediatr Infect Dis J. 1992; 11:88-93.

Farina C,Vailati F, Manisco A, Goglio A. Fungaemia survey:a 10-year experince in Bergamo, Italy. Mycosis. 1999; 42: 543-48.

Favel A, Nguyen AM,Peyron F,Martin C, Thomachot L, Datry A, Bouchara JP, Challier S,Nöel T, Chastin C,Patrick R. Colony morphology switching of 
Candida lusitaniae and acquisition of multidrug resistence during treatment of a renal infection in a newborn: case report and review of the literature. Diagn Microbiol Infect Dis. 2003;47:331-9.

Fernandez M, Moylett EH, Noyola DE, Baker CJ. Candidal Meningitis in Neonates: A 10 -Year Review. Clin Infect Dis. 2000;31:458-63.

Flanagam PG, Barnes RA. Fungal infection in the intensive care unit. $\mathrm{J}$ Hosp Infect. 1998;38:163-77.

Frattanelli DAC, Reed MD, Giacoia GP, Aranda JV. Antifungals in systemic neonatal candidiasis. Drugs. 2004;64:949-68.

Gagneur A, Sizun J, Vernotte E, Parscau L, Quinio D, Flohic AML, Baron R. Low rate of Candida parapsilosis - related colonization and infection in hospitalized preterm infants: a one- year prospective study. J Hosp Infect. 2001;48:193-97.

Gago LC, Capone A, Trese MT. Bilateral presumed endogenous Candida endophthalmitis and stage 3 retinopathy of prematurity. Am J Ophthalmol. 2002;134:611-13. 
Glick C, Graves GR, Feldman S. Neonatal fungemia and amphotericin B. South Med J .1993;86:1368-71.

Guida JD,Kuning AN, Leef KH, Mckenzie SE,Paul DA. Plaquet Count and Sepsis in Very Low Birth Weight Neonates: Is There an organism -specific response?. Pediatrics 2003; 111:1411-15.

Guzmán JM, Jaraba MP, Torre DL,González MDR, Huertas MD, Alvarez R, Zapatero M.Parenteral nutrition and immature neonates. Comparative study of neonates weighing under 1000 and $1000-1250 \mathrm{~g}$ at birth. Early Hum Develop. 2001;65:133-44

Hajjeh RA, Sofair AN, Harrison LH, Lyon M, Arthington-Skaggs BA, Mirza SA, Phelan M, Morgan J, Lee-Yang W, Ciblak MA, Benjamin LE, Sanza T, Huie S, Yeo SF, Brandt ME, Warnock DW. Incidence of bloodstream infections due to candida species and in vitro susceptibilities of isolates collected from 1998 to 2000 in population-dased active surveillance program. J Clin Microbio. 2004;1519-27.

Hitchcock RJI, Pallett A, Hall MA, Malone PSJ. Urinary tract candidiasis in neonates and infants. Brit J Urol. 1995;76:252-6. 
Holler B,Omar SA, Farid MD, Patterson MJ. Effects of Fluid and eletrolyte management on amphotericin B-induced nephrotoxicity among extremely low birth weight infants. Pediatrics. 2004;113:608-16.

Hostetter MK. New insights into candidal infections. Adv Pediatr. 1996; ;209-30.

Huang YC,Lin TY, Lien RI, Chou YH, Kuo CY, Yang PH, Hsieh WS.Candidaemia in Special Care Nurseries: Comparison of Albicans and Parapsilosis Infection. J Infect 2000; 40:171-5.

Huang YC, Kao HT, Lin TY, Kuo AJ. Antifungal susceptibility testing and the correlation with clinical outcome in neonatal candidemia. Am J Perinatol 2001;18:141-6.

Isaacs D, Barfield CP, Grimwood K, McPhee AJ, Minutillo C,Tudehope D. Systemic bacterial and fungal infections in infants in Australian neonatal units. Med J Austr.1995;162:198-201.

Jarlov JO, Born P, Bruun B. Candida albicans Meningitis in a 27 weeks premature infant treated with liposomal amphotericin-B (AmBsome $\left.{ }^{\mathrm{R}}\right)$. Scand J Infect Dis.1995;27:419-20. 
Johnson D, Thompson T, Green T, Ferrierl P. Systemic candidiasis in very low birth weight infants (< 1500 grams). Pediatrics.1984; 73:138-43.

Johnston WT, Cogen MS. Systemic Candidiasis with catarat formation in a premature infant. JAAPOS. 2000;386-8.

Jorge MM, Vázquez AP, Garcia JGS, Ugidos PO, Garcia MPA. Candidiasis cerebral em um recién nacido. An Pediatr. 2003;58:194-195.

Juster RA, Leibovitz E, Linder N , Amitay M, Flidel RO, Even TS, Mogilner B, Barzilai. Liposomal amphotericin B (ambisome) in the treatment of neonatal candidiasis in very low birth weight infants. Infection. 2000;28:223-6.

Juster-Reicher A, Flidel-Rimon O, Amitay M, Even- Tov S, Shinwell E, Leibovitz E. High-dose liposomal amphotericin B in the therapy of systemic candidiasis in neonates. Eur J Clin Microbiol Infect Dis. 2003;22:603-7.

Kamitsuka M, Nugent NA, Conrad PD, Swanson TN. Candida albicans brain abscesses in a premature infant treated with amphotericin $B$, flucytosine and fluconazole. Pediatr Infect Dis J. 1995;329-31.

Kao AS, Brandt ME, Pruitt WR, Conn LA, Perkins BA, Stephens DS, Baughman WS, Reingold AL, Rothrock GA, Pfaller MA, Pinner RW, Hajeh 
RA. The epidemiology of candidemia in two United States cities: results of a population -based active surveillance. Clin Infect Dis.1999; 29:1164-70.

Karlowicz MG, Buescher S, Surka AE. Fulminant late -onset sepsis in a neonatal intensive care unit, 1988-1997, and the impact of avoiding empiric vancomycin therapy. Pediatrics. 2000a;106:1387-90.

Karlowicz MG, Giannone PJ, Pestian J, Morrow AL, Shults J. Does candidemia predict threshold retinopathy of prematurity in extremely low birth weight $(\leq 1000 \mathrm{~g})$ neonates? Pediatrics. 2000b; 105:1036-40.

Karlowicz MG, Rowen JL,Barnes-Eley ML, Lawson ML, Bendel CM et al. The role of birth weight and gestacional age in distinguishing extremely low birth weight infants at high risk of developing candidemia from at low risk: a multicenter study. Pediatr Res. 2002;51:301A.

Karpman E, Kurzrock EA, Low RK. Percutaneous nephroscopic removal of obstructing fungal benzoars and endopyelotomy in an infant. $J$ Urol. 2003;169:1499-500.

Kaufman D, Boyle R, Razen KC, Patrie JT, Robson M, Donowitz LG. Fluconazole prophylaxis against fungal colonization and infection in preterm infants.N.Engl J Med. 2001; 345:1660-66 . 
Kaufman D. Fungal infection in the very low birthweight infant. Curr Opinin infect Dis. 2004;17:253-59.

Kibbler CC,Seaton S, Barnes, Gransden WR, Holliman RE, Johnson EM, Perry JD, Sullivan DJ,Wilson JA. Do so cie i sn $\mathrm{m}$ hanr he i ht dfhmynbio e istir nihsy main $\mathrm{h}$ Candida ypirii $\mathrm{y}$ is Escf os $\mathrm{m}$ osm Wofiy. $\mathrm{J}$ Hosp Infect. 2003;54:18-24.

Kim JO, Garofalo L, Blecker- Shelly D, McGowan K. Candida dubliniensis infections in a pediatric population: retrospective identification from clinical laboratory isolates of candida albicans. J Clin Microbiol. 2003;3354-7.

Klein OJ.Bacterial sepsis and meningitis. In: Remington JS, Klein JO(eds): Infections diseases of the fetus and newborn infant. Philadelphia: WB Saunders; 2001. p. 943-998.

Klingspor L, Stitzing G, Johansen K, Murtaza A, Holmberg K. Infantile diarrhea and malnutrition associated with candida in a developing community. Mycoses. 1993;36:19-24.

Klingspor L, Törnnqvist EWA, Johansson A, Petrini B, Forsum U, Hedin G. A prospective epidemiological survey of candidaemia in sweden. Scand J Infect Dis. 2004; 36:52-5. 
Ko SC, Chen KY, Hsueh PR, Luh KT, Yang PC.Fungal empyema thoracis . an emerging clinical entidy. Chest. 2000;117:1672-78.

Kossoff EH, Buescher ES, Karlowicz MG. Candidemia in a neonatal intensive care unit: trends during fifteen years and clinical features of 111 cases. Pediatr Infect Dis J. 1998;17:504-8.

Kotwani RN, Bodhe PV, Kirodian BG, Mehta KP, Ali US, Kshirsagar NA. Treatment of neonatal candidiasis with liposomaç amphotericin B(L-AMPLRC-1): phase II study. Indian Pediatr. 2003; 40:545-50.

Krebs VLJ, Diniz EMA, Ceccon MEJ, Giolo CRM, Vaz FAC. Lipid formulations of amphotericin B treatment for neonatal systemic candidiasis. In: Proceedings of 2 nd Internacional Congress on New Technologies in Reproductive Medicine Neonatol and Gynecology; 1999a p.527-30.

Krebs VLJ, Pedroso CPA, Diniz EMA, Tamanaha J, Ceccon MEJR, Feferbaum R, Moysés RL,Wagenführ J, Vaz FAC. Endocardite bacteriana como complicação de sepse neonatal-relato de caso. Rev Ass Med Bras. 1999b 45:371-4.

Kremery V, Barnes AJ. Non-albicans Candida spp. causing fungaemia: pathogenicity and antifungal resistence .J Hosp Infect.2002;50:243-60. 
Lackner H, Schwinger W, Urban C, Müller W, Ritschl E, Reiterer F, KuttnigHam M,Urlesberger B, Hauer C. liposomal amphotericin -B (ambisome) for treatment of disseminated fungal infections in two infants of very low birth weight. Pediatrics. 1992;1259-61.

Lee BE, Cheung PY, Robinson JL et al. Comparative study of mortality and morbidity in premature infants ( birth weight $<1250 \mathrm{~g}$ ) with candidemia or candidal meningitis. Clin Infect Dis. 1998;27:559-65.

Leibovitz E. Neonatal candidosis : clinical picture, management controversies and consensus, and new therapeutic options. J Antimicrobial Chemother. 2002;49:69-73.

Linder N, Friling R, Ashkenazi S, Birk E, Snir M, Sirota L. Candidal atrial fungus ball with ocular sequelae. J Pediatr. 2000;137:135.

Linder N, Klinger G, Shalit L, Levy I, Ashkenazi S, Haski, Levit O, Sirota L. Treatment of candidaemia in premature infants:comparison of three amphotericin B preparations. J Antimicrobial Chemother. 2003;52:663-67.

Lunel FMV, Voss A, Kuijper EJ, Gelinck LBS, Hoogerbrugge PM, Liem KL,Kullberg BJ, Verweij PE. detection of candida antigen mannan in cerebrospinal fluid specimens from patients suspected of having candida meningitis. J Clin Microbiol. 2004;867-70. 
Lupetti A, Tavanti A, Davini P, Ghelardi E, Corsini V, Merusi I, Boldrini A, Campa M, Senesi S. Horizontal transmission of candida parapsilosis candidemia in a Neonatal Intensive Care Unit. J Clin Microbiol. 2002;236369.

Lyytikäinen O,LumioJ, Sarkkinen H, Kolho E, Ruutu P. Nosocomial Bloodstream Infections in Finnish Hospitals during 1999-2000. Clin Infect Dis. 2002;35:14-9.

MacDonnell M, Lam AH, Isaacs D. Nonsurgical management of neonatal obstrutive uropathy due to candida albicans. Clin Infect Dis. 1995;21:134950.

Macphail GLP, Taylor GD, Chell B, Ross C, Wilson S, Kureishi A. Epidemiology, treatment and outcome of candidemia: a five-year review at three Canadian hospitals. Mycosis. 2002;45:141-45.

Makhoul IR,Kassis I, Smolkin T, Tamir A, Sujov P. Review of 49 neonates with acquired fungal sepsis: further characterization. Pediatrics. 2001;107:616.

Malani PN, Bradley SF, Little RS, Kauffman CA. Trends in species causing fungaemia in a terciary centre over 12 years. Mycosis. 2001;44:446-49. 
Mazor M,Chaim W, Shinwell ES, Glezerman M. Asymptomatic amniotic fluid invasion with Candida albicans in preterm premature rupture of membranes. Acta Obstet Gynecol Scand.1993;72:52-4.

Mehta R, Weinberger B, Usmani SS, Wapnir RA, Harper RG.Theophylline alters neutrophil function in preterm infants. Biol Neonate. 2002;81:176-81.

Miller MJ:Fungal infections. In: Remington JS, Klein JO(eds): Infections diseases of the fetus and newborn infant. Philadelphia: WB Saunders; 2001. p.813-39.

Moise A, Landers S, Fraley K.Colonization and infection of umbilical catheters in newborn infants .Pediatr Res 1986; 20:1436A.

Moreno MT,Vargas S, Poveda R, Sáezllorens X. Neonatal sepsis and meningitis in a developing Latin American country. Pediatr Infect Dis $\mathrm{J}$. $1994 ; 13: 516-20$

Murphy K, Bradley J, James HE .The treatment of Candida albicans shunt infections. Child's Nerv Syst. 2000;16:4-7.

Ng PC. Systemic fungal infections in neonates. Arch Dis Child 1994;71:13035. 
Ng PC, Lee CH,Fok TF, Chui K, Wong W, Cheung KL, So KW. Central nervous system candidiasis in preterm infants: Limited value of biochemical markers for diagnosis. J Pediatr Child Health. 2000; 36:509-10.

Noyola DE, Fernandez M, Moylett EH, Baker CJ . Ophthalmologic, visceral, and cardiac involvement in neonates with candidemia. Clin Infect Dis. $2001 ; 32: 1018-23$

Noyola DE, Bohra L,Paysse EA, Fernandez M, Coats DK. Association of candidemia and retinopathy of prematurity in very low birthweight infants. Ophthalmology. 2002;109:80-4.

Paganini H, Brieshcke TR, Santos P, Séu S, Rosanova MT. Risk factors for nosocomial study in children .J Hosp infect. 2002;50:304-08.

Patrick CC. Infection in. New York: Churchill Livingstone; 1992. p:539-50: Candida.

Perroni JA.Laryngeal obstruction due to Monilia albicans in a newborn. Laryngoscope. 1970;80:228-91.

Rabalais GP, Samiec TD, Bryant KK, Lewis JJ. Invasive candidiasis in infants weighting more than 2500 grams at birth admitted to a neonatal intensive care unit. Pediatr Infect Dis J.1996;15:348-52. 
Rennert G, Rennert HS, Pitlik S, Finkelstein R, Kitzes CR. Epidemiology of candidemia a nationwide survey in Israel. Infection. 2000;28:26-9.

Reyes MCR, Gutiérrez FF, Dominguez P, et al.Candidiasis sistémica neonatal en los noventa. An Pediatr. 1996;44:257-61.

Richet HM, Andremont AQ,Tancrede, Pico JL, Jarvis W. Risk factors for candidaemia in pacients with acute lymphocytic leukemia. Rev Infect Dis.1991;13:211-15.

Rios AP,Hernández LA, Cruz MC, Dominguez GS, Figueroa CA, Santos Jl. Endocarditis por candida en el primer año de vida. Bol Med Hosp Infant Mex. $1993 ; 157-61$

Robertson NJ, Kuna J, Cox PM, Lakhoo K. Spontaneous intestinal perforation and candida peritonitis presenting as extensive necrotizing enterocolitis. Acta Paediatr. 2003;92:258-64.

Roilides E, Farmaki E, Evdoridou J,Francesconi A, Kasai M, Filioti J, Tsivitanidou M, Sofianou D, Kremenopoulus G, Wash T. candida tropicalis in a neonatal intensive care unit :epidemiologic and molecular analysis of na outbreak of infection with na uncommon neonatal pathogen. J Clin Microl. $2003 ; 735-41$ 
Rowen JL, Rench MA, Kazinetz CA, Adams Jr JM, Baker CJ. Endotracheal colonization with Candida enhances risk of systemic candidiasis in very low birth weight neonates. J Pediatr. 1994;124:789-94.

Safdar A, Bannister TW, Safdar Z.The predictors of outcome in immunocompetent pacients with hematogenous candidasis. Int J Infect Dis. 2004;8:180-6.

Saiman L, Ludington $\mathrm{E}$, Pfaller $\mathrm{M}$, et al. Risk factors for candidemia in neonatal intensive care unit pacients. The national epidemology of mycosis survey study group. Pediatr.Infect Dis J. 2000;19:319:24.

Salzman BM, Isenberg HD, Shapiro JF, Lipsitz PJ, Rubin LG . A prospective study of the catheter hub as the portal of entry for microorganisms causing catheter-related sepsis in neonates. J Infect Dis. 1993;167:487-90.

Sanchez PJ, Siegel JD, Fishbein J. Candida endocarditis: successful medical manegement in three preterm infants and review of the literature. Pediatr Infect Dis J 1991; 10: 239-43.

Sastre JBL, Cotallo GDC, Colomer BF, Castrillo GH. Neonatal Invasive Candidiasis: A Prospective Multicenter Study of 118 Cases. Am J perinatol. 2003;153-63. 
Sax $\mathrm{H}$, Hugonnet S, Harbarth S, Herrault P, Pittet D. Variation in nosocomial infection prevalence according to pacient care setting:a hospital-wide survey. J Hosp Infect. 2001;48:27-32.

Saxen H, Virtanen M, Carlson P, Hoppu K, Pohjavuori M, Vaara M, Varkila V, Peltola $\mathrm{H}$. Neonatal candida parapsilosis outbreak with a highcase fatality rate. Pediatr Infect Dis J. 1995;14:776-81.

Scarcella A, Pasquariello MB, Giugliano B, Vendemmia M, De Lucia A. Liposomal Amphotericin B treatment for neonatal fungal infections. Pediatr Infect Dis J. 1998;17:146-8.

Sigmundsdóttir G, Christensson B, Björklund LJ et al.Urine-arabinitol/ Larabinitol ratio in diagnosis of invasive candidiasis in newborn infants. $J$ Clin Microbiol 2000; 38:3.039-42.

Silva CLP,Santos RMR,Colombo AL.Cluster of Candida parapsilosis primary bloodstream infection in a neonatal intensive care unit. Braz $\mathrm{J}$ Infect Dis $2001 ; 5: 32-6$.

Stern JH, Calvano C, Simon JW. Recurrent Endogenous candidal endophthalmitis in a premature infant. JAAPOS. 2001;5:50-1. 
Stocker M, Caduff JH,Spalinger J, Berger TM. Successful treatment of bilateral renal fungal balls with liposomal amphotericin B and fluconazole in a extremely low birth weight infant. Eur J Pediatr. 2000;159:676-78.

Stoll BJ, Gordon T,Korones SB,Shankaran S, Tyson JE,Bauer C, et al.Lateonset sepsis in very low birth weight neonates: a report from the National Institute of child health and human development neonatal research network . J Pediatr.1996;29:63-71.

Stoll BJ, Hansen N, Fanaroff AA, Wright LL,Carlo WA,Ehrenkranz RA, Lemons JA et al. Late- onset sepsis in very low birth weight neonates:the experience of the NICHD Neonatal Research Network. Pediatrics. 2002; $110: 285-91$

Strong M, Lejman T, Michno P. Septic arthritis of wrist in infancy. J Pediatr Orthop.1995;15:152-6.

Tirodker UH, Nataro JP, Smith S, Las Casas L, Fairchild KD. Detection of fungemia by polymerase chain reaction in critically ill neonates and children. J Perinatol 2003, 23:177-22. 
Tortorano AM, Biraghi E, Astolfi A, Ossi C, Farina C, Perin S, Bonaccorso C, Cavanna C, Raballo A, Grossi A, FIMUA Candidemia study group. J Hosp Infect. 2002;51:297-304.

Vricella LA, Khambadkone S, Yates R, Tsang VT. Right ventricular inflow obstruction from massive fungal vegetation presenting as neonatal circulatory collapse. Eur J Cardio-thoracic Surg. 2003;24:323-4.

Vaideeswar,P, Deshpande J, Sivaraman A. Isolated left-sided intracardiac fungal mass. Int J cardiol. 1994;43:97-8.

Walsh TJ, Merz WG, Lee JW et al. Diagnosis and therapeutic monotoring of invasive candidíasis by rapid enzimatic detection of serum D-arabinitol. Am J Med 1995; 99:164-71.

Warady BA, Bashir M, Donaldson LA. Fungal peritonitis in children receiving peritoneal dialysis: A report of the NAPRTCS. Kidney Intern. 2000;58:384-9.

Warris A, Semmekrot A, Voss A. Candidal and bacterial bloodstream infections in prematures neonates: a case- control study. Med Mycol. 2001;39:75-9. 
Weese-Mayer DE, Fondriest DW, Brouillete RT, Shulman ST .Risk factors associated with candidemia in the neonatal intensive care unit: a casecontrol study. Pediatr Infect Dis J. 1987;6:190-6.

Weintrub PS,Chapman A, Piecuch R. Renal Fungus Ball in a premature infant successfully treated with fluconazole. Pediatr Infect Dis $\mathrm{J}$. $1994 ; 13: 1152-54$.

Weitkamp JH, Poets CF, Sievers et al.: Candida infection in very low birth weight infants: outcome and nephrotoxicity of treatment with liposomal amphotericin B(AmBisome). Infection.1998; 26:11-5.

Wey S, Motoni M, Pfaller MA, Woolson RF, Wenzel RP. Risk factors for hospital-acquired candidaemia . A matched case-control study. Arch Intern Med. 1989;149:2349-53.

Wimalendra M, Reece A, Nicholl RM.Renal fungal ball. Arch Dis Child Fetal Neonatal. 2004;89:376.

Winter JTV,Ney JA,Ogburn PL,Johnson RV. Preterm labor and congenital candidiasis. a case report. J Reprod Med. 1994;39:987-90. 
Winters WD, Shaw DWW, Weinberger E. Candida Fungus Balls Presenting as Intraventricular Masses in Cranial Sonography.J Clin Ultrasound. 1995;23:266-70.

Witek-Janusek L, Shareef MJ,Mathews HL. Reduced lymphocyte- mediated antifungal capacity in high-risk infants. J Infect Dis. 2002;186:129-33.

Yamamura DLR, Rotstein C, Nicolle LE, Ioannou S. Candidemia at selected Canadian sites:results from the fungal disease registry,1992-1994. CMAJ $1999 ; 160: 93-9$

Yinnon M, Woodin KA,Powell KR. Candida lusitaniae infection in the newborn :case report and review of the literature. Pediatr Infect Dis $\mathrm{J}$. $1992 ; 11: 878-80$.

Yoo SY, MKN Wonju, Korea. Acute renal failure caused by fungal benzoar: a late complication of candida sepsis associated with central catheterization. $J$ Pediatr Surg. 1995; 30:1600-02. 